\title{
Periods and points attached to quadratic algebras
}

\author{
Massimo Bertolini \\ Henri Darmon \\ Peter Green
}

September 5, 2007

\section{Contents}

1 Classical Heegner points 4

2 Heegner points and $p$-adic integration $\quad 9$

3 Forms on $\mathcal{T}_{p} \times \mathcal{H} \quad 23$

4 Complex periods and Heegner points $\quad 27$

$5 \quad p$-adic periods and Stark-Heegner points 33

6 Heegner points and integration on $\mathcal{H}_{p} \times \mathcal{H}_{q} \quad 40$

7 Periods of Hilbert modular forms 48

\section{Introduction}

Let $E$ be an elliptic curve over $\mathbb{Q}$ of conductor $N$. By the fundamental work initiated in [Wi] and brought to a successful conclusion in [BCDT], the curve $E$ is equipped with a non-constant complex uniformisation

$$
\Phi: \mathcal{H} / \Gamma_{0}(N) \longrightarrow E(\mathbb{C})
$$


where $\mathcal{H}$ is the Poincaré upper half-plane and $\Gamma_{0}(N)$ is Hecke's congruence group of level $N$.

Fix a quadratic field $K$; when it is imaginary, the theory of complex multiplication combined with (1) yields the construction of a remarkable collection of points on $E$ defined over certain ring class fields of $K$. These are the Heegner points recalled in section 1 whose study is one of the themes of this proceedings volume.

When $p$ is a prime that divides $N$ exactly, and $E$ a factor of the Jacobian of a Shimura curve attached to a quaternion algebra ramified at $p$, the uniformisation $\Phi$ of (1) admits a $p$-adic analogue based on theorems of Jacquet-Langlands and Cerednik-Drinfeld, in which $\mathcal{H}$ is replaced by Drinfeld's $p$-adic upper half plane $\mathcal{H}_{p}:=\mathbb{C}_{p}-\mathbb{Q}_{p}$, and $\Gamma_{0}(N)$ by an appropriate $\{p\}$-arithmetic subgroup of a definite quaternion algebra over $\mathbb{Q}$. This somewhat less well-known theory is recalled in section 2 , where a $p$-adic variant of the complex Heegner point construction of section 1 is described.

Retaining the assumption that $N=p M$ with $p \nmid M$, section 3 introduces the $\{p\}$-arithmetic group $\Gamma \subset \mathbf{S L}_{2}(\mathbb{Q})$ defined by

$$
\Gamma=\left\{\left(\begin{array}{ll}
a & b \\
c & d
\end{array}\right) \in \mathbf{S L}_{2}(\mathbb{Z}[1 / p]) \text { such that } M \text { divides } c\right\} \text {. }
$$

This group acts both on $\mathcal{H}$ and on $\mathcal{H}_{p}$ by Möbius transformations, and the diagonal action of $\Gamma$ on $\mathcal{H}_{p} \times \mathcal{H}$ is discrete. A double integral on $\left(\mathcal{H}_{p} \times \mathcal{H}\right) / \Gamma$ is defined to be a $\mathbb{C}$ or $\mathbb{C}_{p}$-valued function of $\left(\tau_{1}, \tau_{2}, \tau_{3}, \tau_{4}\right) \in \mathcal{H}_{p}^{2} \times \mathcal{H}^{2}$, denoted

$$
\int_{\tau_{1}}^{\tau_{2}} \int_{\tau_{3}}^{\tau_{4}} \omega_{f} \in \mathbb{C} \text { or } \mathbb{C}_{p}
$$

satisfying the obvious additivity properties suggested by the notation, as well as being invariant under the diagonal action of $\Gamma$. The normalised eigenform $f$ of weight 2 attached to $E$ is shown in section 3 to give rise in a natural way to a $\mathbb{C}$-valued double integral on $\left(\mathcal{H}_{p} \times \mathcal{H}\right) / \Gamma$. This function can be viewed as encoding the complex periods of a "form $\omega$ of weight $(2,2)$ " on $\left(\mathcal{H}_{p} \times \mathcal{H}\right) / \Gamma$ attached to $E$.

Section 4 reformulates the results of section 1 in terms of the $\mathbb{C}$-valued double integral attached to $f$. This amounts to revisiting the classical theory from a slightly different perspective, in which the first cohomology of $\Gamma_{0}(N)$ is replaced by the second cohomology of $\Gamma$. The Heegner points obtained in this construction are the same as those of section 1. In particular, they are 
defined over the ring class fields of a quadratic field $K$ in which the place $\infty$ is inert (i.e., $K$ is imaginary quadratic) and the place $p$ is split.

The main advantage of the (at first sight overly recundite) point of view developed in sections 3 and 4 is that the places $\infty$ and $p$ play more symmetrical roles in its formulation. Building on the techniques of section 2 and using a theorem of Manin-Drinfeld already discussed for a different purpose in section 4 , a $\mathbb{C}_{p}$-valued double integral is attached to $f$ in section 5 , leading to a theory in which the roles of $p$ and $\infty$ are interchanged. The manipulations carried out in section 4 make sense in this new context, at least formally, suggesting the possibility of using the $\mathbb{C}_{p}$-valued double integral to construct algebraic points on $E$ defined over ring class fields of a quadratic field $K$ in which $p$ is inert and the prime $\infty$ is split. Because such a $K$ is real quadratic, the resulting construction falls outside the scope of the theory of complex multiplication. For this reason the conjectures of section 5 have so far eluded proof. But they are well-suited to numerical verifications, and can be used to calculate "Heegner points attached to real quadratic fields" [DG] in much the same way that Stark's conjecture can be used to approximate units in certain class fields by analytic means.

Section 6 transposes the theory of section 5 to the product $\mathcal{H}_{p} \times \mathcal{H}_{q}$ of two non-archimedean upper half planes. Here the situation is better understood, and the counterpart of the main conjectures of section 5 can be proved entirely as a consequence the Cerednik-Drinfeld theory presented in section 2. The relative ease in obtaining the results of section 6 indicates that the mystery in section 5 is intimately tied to the presence of an archimedean place (and only accessorily to that of a $p$-adic place) in the product of upper half planes on which the relevant integration theory is defined.

Section 7 reinforces this impression by modifying the setting of section 5 in a different direction, replacing $\mathcal{H}_{p} \times \mathcal{H}$ by the product $\mathcal{H} \times \mathcal{H}$ of two archimedean upper half-planes, and the group $\Gamma$ by a Hecke-type congruence subgroup of the Hilbert modular group $\mathbf{S L}_{2}\left(\mathcal{O}_{F}\right)$. Here $F$ is a real quadratic field and $\mathcal{O}_{F}$ its ring of integers. The role of the double integrals on $\left(\mathcal{H}_{p} \times \mathcal{H}\right) / \Gamma$ is played by the periods of a Hilbert modular form of weight $(2,2)$ which is conjecturally attached to an elliptic curve over $F$. Such period integrals have already been defined and studied extensively in the classical literature. Yet the natural analogues of the conjectures of section 5 in the Hilbert modular form setting have not been studied before and appear to transcend the classical theory of complex multiplication (as well as its generalisation to totally real fields proposed by Hilbert and his school, 
and rigorously developed by Shimura and Taniyama in the 50s and 60s). The article closes by proposing a conjecture which would allow the analytic construction of algebraic points on an elliptic curve $E / F$, points whose coordinates are defined over ring class fields of a quadratic extension $K$ of $F$ with two real and one complex place. A notable feature of this conjecture is that it provides a "modular" construction of algebraic points on an elliptic curve having everywhere good reduction over $F$, although such a curve is not expected in general to appear in the Jacobian of any modular or Shimura curve.

\section{Classical Heegner points}

Let $N$ be a positive integer as in the introduction, and let $X_{0}(N)$ be the modular curve classifying pairs $\left(\mathcal{E}, \mathcal{E}^{\prime}\right)$ of generalized elliptic curves equipped with a cyclic $N$-isogeny $\mathcal{E} \rightarrow \mathcal{E}^{\prime}$. Let $\mathcal{H}^{*}:=\mathcal{H} \cup \mathbb{P}_{1}(\mathbb{Q})$ denote the extended upper half plane and let $\Gamma_{0}(N)$ be the Hecke congruence group of level $N$, acting on $\mathcal{H}^{*}$ by Möbius transformations. There is a well-known identification of Riemann surfaces

$$
j: \mathcal{H}^{*} / \Gamma_{0}(N) \stackrel{\sim}{\longrightarrow} X_{0}(N)(\mathbb{C}),
$$

in which $\tau \in \mathcal{H}$ is mapped to the pair of elliptic curves $(\mathbb{C} /\langle\tau, 1\rangle, \mathbb{C} /\langle\tau, 1 / N\rangle)$ related by the obvious cyclic $N$-isogeny.

Let $\mathcal{O}$ be an order in an imaginary quadratic subfield $K$ of $\mathbb{C}$. Suppose $N$ satisfies the Heegner hypothesis relative to $\mathcal{O}$, so that the ideal $N \mathcal{O}$ factors as a product $N \mathcal{O}=\mathcal{N} \overline{\mathcal{N}}$ of cyclic ideals of norm $N$. Let

$$
\delta: \operatorname{Pic}(\mathcal{O}) \rightarrow X_{0}(N)(\mathbb{C})
$$

be the natural map which to the class of a fractional ideal $\mathfrak{a}$ associates the pair $\left(\mathbb{C} / \mathfrak{a}, \mathbb{C} / \mathcal{N}^{-1} \mathfrak{a}\right)$ of $N$-isogenous elliptic curves.

Write $H$ for the ring class field attached to $\mathcal{O}$, so that the Artin reciprocity map of class field theory induces an identification

$$
\operatorname{rec}: \operatorname{Pic}(\mathcal{O}) \stackrel{\sim}{\rightarrow} \operatorname{Gal}(H / K) .
$$

The maps $\delta$ and rec are related by the fundamental Shimura reciprocity law: 
Proposition 1.1 For any $\mathfrak{a} \in \operatorname{Pic}(\mathcal{O})$, the point $\delta(\mathfrak{a})$ belongs to $X_{0}(N)(H)$. More precisely, for all $\mathfrak{b} \in \operatorname{Pic}(\mathcal{O})$,

$$
\delta\left(\mathfrak{b}^{-1} \mathfrak{a}\right)=\operatorname{rec}(\mathfrak{b}) \cdot \delta(\mathfrak{a})
$$

Sketch of Proof. The set of Heegner points with endomorphism ring equal to $\mathcal{O}$ forms a $\operatorname{Pic}(\mathcal{O})$-affine space via the rule

$$
\mathfrak{a} *\left(\mathcal{E}, \mathcal{E}^{\prime}\right)=\left(\operatorname{Hom}(\mathfrak{a}, \mathcal{E}), \operatorname{Hom}\left(\mathfrak{a}, \mathcal{E}^{\prime}\right)\right), \quad \mathfrak{a} \in \operatorname{Pic}(\mathcal{O}), \quad\left(\mathcal{E}, \mathcal{E}^{\prime}\right) \in X_{0}(N),
$$

which on the level of complex tori is given by

$$
\mathfrak{a} *\left(\mathbb{C} / \Lambda, \mathbb{C} / \mathcal{N}^{-1} \Lambda\right)=\left(\mathbb{C} / \mathfrak{a}^{-1} \Lambda, \mathbb{C} / \mathfrak{a}^{-1} \mathcal{N}^{-1} \Lambda\right) .
$$

Since there are finitely many Heegner points on $X_{0}(N)$ attached to $\mathcal{O}$ and since they are preserved under the action of $\operatorname{Aut}(\mathbb{C})$, they are algebraic. Furthermore, the action of $\operatorname{Gal}(\bar{K} / K)$ on these points commutes with the action of $\operatorname{Pic}(\mathcal{O})$ defined in $(2)$, and hence is described by a homomorphism

$$
\tilde{\delta}: \operatorname{Gal}(\bar{K} / K) \rightarrow \operatorname{Pic}(\mathcal{O}) \text { satisfying } \tilde{\delta}(\sigma) *\left(\mathcal{E}, \mathcal{E}^{\prime}\right)=\left(\mathcal{E}^{\sigma}, \mathcal{E}^{\prime \sigma}\right),
$$

for all Heegner points $\left(\mathcal{E}, \mathcal{E}^{\prime}\right)$ with $\operatorname{End}(\mathcal{E})=\mathcal{O}$. This already shows that the Heegner points are defined over an abelian extension, namely the extension $\tilde{H}=\bar{K}^{\operatorname{ker}(\tilde{\delta})}$ cut out by $\tilde{\delta}$. Now let $\mathfrak{p}$ be a prime of $\mathcal{O}$ unramified in $\tilde{H}$, let $\mathfrak{P}$ be a prime of $\tilde{H}$ above $\mathfrak{p}$ and assume $\mathcal{E}$ has good reduction at $\mathfrak{P}$. A case by case verification (cf. [Se1]) shows that the elliptic curve obtained by reducing $\mathcal{E}(\bmod \mathfrak{P})$ and raising its coefficients to the $\#\left(\mathcal{O}_{K} / \mathfrak{p}\right)$-power is isomorphic to $\operatorname{Hom}(\mathfrak{p}, \mathcal{E})$ reduced $(\bmod \mathfrak{P})$. Hence,

$$
\tilde{\delta}\left(\operatorname{Frob}_{\mathfrak{p}}\right)=[\mathfrak{p}] \in \operatorname{Pic}(\mathcal{O}),
$$

so that, by the Artin reciprocity law, $\tilde{\delta}^{-1}=\operatorname{rec}$. Thus $\tilde{H}=H$, and the result follows. For more details see [Se1].

While CM points on $X_{0}(N)$ are in natural correspondence with elements of Picard groups of orders in imaginary quadratic fields, for more general types of modular curves such as the Shimura curves introduced in section 2 , it is only the $\operatorname{Pic}(\mathcal{O})$-affine action which generalizes. We therefore make a shift in notation, replacing $\operatorname{Pic}(\mathcal{O})$ with the $\operatorname{Pic}(\mathcal{O})$-set $\operatorname{Emb}\left(\mathcal{O}, M_{0}(N)\right)$, defined below. Here $M_{0}(N)$ denotes the order of matrices in $M_{2}(\mathbb{Z})$ which are upper triangular modulo $N$. At first this seems to only add opacity to the 
discourse, but in fact the notation allows greater flexibility which is useful in the sequel.

There is a convenient dictionary between classes of projective modules of rank 1 over $\mathcal{O}$ and embeddings $\Psi$ of the quadratic imaginary field $K \subset \mathbb{C}$ into the central simple algebra $M_{2}(\mathbb{Q})$ satisfying some additional technical hypotheses:

1. The embedding $\Psi$ is said to be optimal (relative to $N$ and $\mathcal{O}$ ) if $\Psi(K) \cap$ $\mathrm{M}_{0}(N)=\Psi(\mathcal{O})$.

2. An orientation of the Eichler order $\mathrm{M}_{0}(N)$ is a surjective ring homomorphism $\mathrm{M}_{0}(N) \rightarrow \mathbb{Z} / N \mathbb{Z}$. One could for instance choose the map

$$
\left(\begin{array}{ll}
a & b \\
c & d
\end{array}\right) \mapsto a(\bmod N)
$$

Then $\Psi$ is said to be oriented (relative to $\mathcal{N}$ and the chosen orientation on $\left.M_{0}(N)\right)$ if the following diagram commutes:

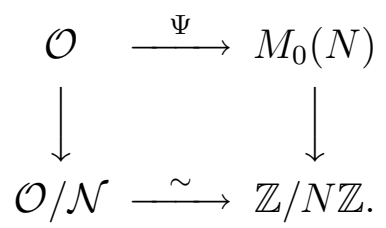

3. There is a unique fixed point $\tau_{\Psi} \in K$ for the action of the group $\Psi\left(K^{\times}\right)$ by Möbius transformations with the property that

$$
\Psi(\lambda)\left(\begin{array}{c}
\tau_{\Psi} \\
1
\end{array}\right)=\lambda\left(\begin{array}{c}
\tau_{\Psi} \\
1
\end{array}\right)
$$

The embedding $\Psi$ is said to be oriented at $\infty$ if $\tau_{\Psi}$ belongs to $\mathcal{H}$, under the fixed embedding of $K$ into $\mathbb{C}$.

An embedding satisfying the three conditions above is called an oriented optimal embedding of $\mathcal{O}$ into $M_{0}(N)$. Note that this notion depends on the fixed choice of the ideal $\mathcal{N}$ and of the orientation on $M_{0}(N)$, as well as on the fact that $K$ is given as a subfield of $\mathbb{C}$.

If $R$ is any subring of a matrix or quaternion algebra, denote by $R_{1}^{\times}$the group of elements in $R$ of (reduced) norm 1 . The reader may check that the group $\Gamma_{0}(N)=M_{0}(N)_{1}^{\times}$acts naturally on the set of oriented optimal 
embeddings by conjugation. Let $\operatorname{Emb}\left(\mathcal{O}, M_{0}(N)\right)$ denote the set of $\Gamma_{0}(N)$ conjugacy classes of oriented optimal embeddings of $\mathcal{O}$ into $M_{0}(N)$.

If $\Psi$ is an oriented optimal embedding of $\mathcal{O}$ into $M_{0}(N)$, equation (3) shows that the lattice $\mathbb{Z} \tau_{\Psi} \oplus \mathbb{Z} \subset \mathbb{C}$ is preserved under multiplication by $\mathcal{O}$; the optimality of $\Psi$ implies that it is in fact a projective $\mathcal{O}$-module. Let $\mathfrak{a}_{\Psi}$ denote its class in $\operatorname{Pic}(\mathcal{O})$. Replacing $\Psi$ by an embedding that is conjugate to it under $\Gamma_{0}(N)$ does not change the class $\mathfrak{a}_{\Psi}$, and hence the assignment $\Psi \mapsto \mathfrak{a}_{\Psi}$ is a well defined function from $\operatorname{Emb}\left(\mathcal{O}, M_{0}(N)\right)$ to $\operatorname{Pic}(\mathcal{O})$.

Lemma 1.2 The assignment $\Psi \mapsto \mathfrak{a}_{\Psi}$ is a bijection between $\operatorname{Emb}\left(\mathcal{O}, M_{0}(N)\right)$ and $\operatorname{Pic}(\mathcal{O})$.

Proof. Suppose that $\mathfrak{a}$ belongs to $\operatorname{Pic}(\mathcal{O})$. The class $\mathfrak{a}$ has a representative of the form $\left\langle\tau_{\mathfrak{a}}, 1\right\rangle$, with $\tau_{\mathfrak{a}} \in \mathcal{H}$, such that

$$
\mathcal{N}^{-1}\left\langle\tau_{\mathfrak{a}}, 1\right\rangle=\left\langle\tau_{\mathfrak{a}}, 1 / N\right\rangle .
$$

Furthermore, this $\tau_{\mathfrak{a}}$ is determined by $\mathfrak{a}$ up to the action of $\Gamma_{0}(N)$. We may define an optimal embedding $\Psi_{\mathfrak{a}}: K \hookrightarrow M_{2}(\mathbb{Q})$ by setting

$$
\Psi_{\mathfrak{a}}\left(\tau_{\mathfrak{a}}\right)=\left(\begin{array}{cc}
\operatorname{Tr}\left(\tau_{\mathfrak{a}}\right) & -\mathrm{Nm}\left(\tau_{\mathfrak{a}}\right) \\
1 & 0
\end{array}\right)
$$

whose class in $\operatorname{Emb}\left(\mathcal{O}, M_{0}(N)\right)$ depends only on $\mathfrak{a}$ and not on the choice of ideal representative. The condition (4) forces the embedding $\Psi_{\mathfrak{a}}$ to be oriented. The reader will check that assignment $\mathfrak{a} \mapsto \Psi_{\mathfrak{a}}$ defines a map $\operatorname{Pic}(\mathcal{O}) \rightarrow \operatorname{Emb}\left(\mathcal{O}, M_{0}(N)\right)$, which is inverse to the map $\Psi \mapsto \mathfrak{a}_{\Psi}$. This proves the lemma.

Thanks to lemma 1.2, the regular action of $\operatorname{Pic}(\mathcal{O})$ on itself by left multiplication translates into an action of this group on $\operatorname{Emb}\left(\mathcal{O}, M_{0}(N)\right)$, which we denote

$$
(\mathfrak{a}, \Psi) \mapsto \Psi^{\mathfrak{a}}
$$

This action can be described adelically as follows. Given $\mathfrak{a} \in \operatorname{Pic}(\mathcal{O})$, let $a$ be the finite idele of $K$ which corresponds to it by the rule $a(\mathcal{O} \otimes \hat{\mathbb{Z}})=\mathfrak{a} \otimes \hat{\mathbb{Z}}$. One can assume that $\operatorname{Norm}_{\mathbb{Q}}^{K}(a) \in \mathbb{Q}^{\times} \subset \mathbb{A}_{\mathbb{Q}}^{f \times}$. By strong approximation, there is an element $\gamma \in \mathrm{GL}_{2}(\mathbb{Q})$ such that

$$
\gamma=\Psi(a) u \quad \text { with } u \in\left(M_{0}(N) \otimes \hat{\mathbb{Z}}\right)_{1}^{\times} .
$$

(Here $\Psi$ has been extended in the natural way to a map $\mathbb{A}_{K}^{f} \rightarrow M_{2}\left(\mathbb{A}_{\mathbb{Q}}^{f}\right)$, still denoted by $\Psi$.) 
Lemma 1.3 For any $\gamma$ satisfying (5),

$$
\Psi^{\mathfrak{a}}=\gamma^{-1} \Psi \gamma
$$

As in the introduction, let $E / \mathbb{Q}$ be an elliptic curve of conductor $N$, equipped with a modular parametrisation of minimal degree

$$
\Phi: X_{0}(N) \rightarrow E
$$

mapping the cusp $\infty$ to the identity of $E$. Let $\Lambda_{E}$ be the lattice generated by the periods of a Néron differential $\omega_{E}$ on $E$, and let

$$
\eta: \mathbb{C} / \Lambda_{E} \rightarrow E(\mathbb{C})
$$

be the Weierstrass uniformisation attached to $\Lambda_{E}$, or, equivalently, to the choice of differential $\omega_{E}$. (Cf. [Si], ch. VI.5.) Up to a non-zero rational constant - the Manin constant, which is expected to be \pm 1 , a fact which will be assumed from now on - the pull-back $(\Phi \circ j)^{*} \omega_{E}$ is equal to $2 \pi i f(t) d t$ where

$$
f=\sum_{n=1}^{\infty} a_{n} e^{2 \pi i n \tau}
$$

is the normalized weight 2 cusp form attached to $E$. Suppose $\tau \in \mathcal{H}$ is such that $\mathcal{N}^{-1}\langle\tau, 1\rangle$ equals $\langle\tau, 1 / N\rangle$ up to homothety. Then the results of [Si], ch. VI.5 reveal that

$$
\Phi(\delta(\langle\tau, 1\rangle))=\eta\left(\int_{i \infty}^{\tau}(\Phi \circ j)^{*} \omega_{E}\right) .
$$

Given an embedding $\Psi$ of $K$ into $M_{2}(\mathbb{Q})$, we may define a period

$$
J_{\Psi}:=\int_{i \infty}^{\tau_{\Psi}} 2 \pi i f(t) d t=\sum_{n=1}^{\infty} \frac{a_{n}}{n} e^{2 \pi i n \tau_{\Psi}} .
$$

where $\tau_{\Psi} \in \mathcal{H}$ is the fixed point attached to $\Psi$ as earlier. Restating equation (6), the class $\mathfrak{a}_{\Psi} \in \operatorname{Pic}(\mathcal{O})$ of $\left\langle\tau_{\Psi}, 1\right\rangle$ satisfies $\Phi\left(\delta\left(\mathfrak{a}_{\Psi}\right)\right)=\eta\left(J_{\Psi}\right)$. Since the map $\Phi$ is defined over $\mathbb{Q}$, the following is a direct consequence of proposition 1.1:

Theorem 1.4 For all $\Psi \in \operatorname{Emb}\left(\mathcal{O}, M_{0}(N)\right)$, the point $\eta\left(J_{\Psi}\right)$ belongs to $E(H)$, and for all $\mathfrak{b} \in \operatorname{Pic}(\mathcal{O})$,

$$
\eta\left(J_{\Psi^{\mathfrak{b}}}\right)=\operatorname{rec}(\mathfrak{b})^{-1} \cdot \eta\left(J_{\Psi}\right) .
$$


This proposition asserts that the analytically defined invariants $J_{\Psi}$ of equation (7) map under $\eta$ to a system of algebraic points on the associated elliptic curve. It is exactly this phenomenon (stripped of the algebro-geometric underpinnings provided by proposition 1.1) that we seek to generalize in this article.

Accordingly, the next sections introduce more general types of periods attached to the following data:

1. an embedding of a quadratic algebra $K$ into a quaternion algebra $B$;

2. a cusp form on $B$ attached to an elliptic curve $E$;

3. an appropriate integration theory on a product of (archimedean and non-archimedean) upper half-planes.

The images of such periods under the appropriate (Weierstrass or Tate) uniformisation are expected to yield a system of algebraic points on $E$ which behave just like the system of Heegner points defined in this section, insofar as

1. they satisfy a variant of the Shimura reciprocity law, and

2. their heights are related to derivatives of Rankin $L$-series attached to $E$ and $K$ in the spirit of the classical Gross-Zagier formula.

This expectation can be proved by means of the theory of complex multiplication in the cases considered in section 2,4 and 6 ; it is only conjectural in the cases of section 5 and 7 .

\section{Heegner points and $p$-adic integration}

This section explains a construction of generalised Heegner points arising from certain Shimura curve parametrisations, in terms of $p$-adic period integrals. Frequently this construction can be performed on the modular elliptic curve $E$ considered in section 1 . 


\section{Forms on $\mathcal{H}_{p}$}

Suppose in this section that the conductor $N$ of $E$ admits an integer factorisation of the form

$$
N=p N^{+} N^{-},
$$

where $p$ is prime, the three factors are pairwise relatively prime, and $N^{-}$is a squarefree integer divisible by an odd number of primes. In particular, $E$ has multiplicative reduction at $p$.

Write $B$ for the (definite) quaternion algebra over $\mathbb{Q}$ of discriminant $N^{-}$ and $R$ for an Eichler $\mathbb{Z}[1 / p]$-order of $B$ of level $N^{+}$. The order $R$ is unique up to conjugation by elements of $B^{\times}$. (For more on the arithmetic theory of quaternion algebras, see [Vi].)

As in the introduction, let $\mathcal{H}_{p}$ denote Drinfeld's $p$-adic upper half plane. It is equal to $\mathbb{P}_{1}\left(\mathbb{C}_{p}\right)-\mathbb{P}_{1}\left(\mathbb{Q}_{p}\right)=\mathbb{C}_{p}-\mathbb{Q}_{p}$, and carries a natural structure of a rigid analytic space. The rigid analytic functions on $\mathcal{H}_{p}$ play the role in this discussion of holomorphic functions on $\mathcal{H}$; since this notion is so important for the sequel we recall its precise definition which requires the introduction of certain attendant structures on $\mathcal{H}_{p}$.

Let $\mathcal{T}_{p}$ be the Bruhat-Tits tree of $\mathbf{P G} \mathbf{L}_{2}\left(\mathbb{Q}_{p}\right)$. It is a homogeneous tree of degree $p+1$, whose set $\mathcal{V}\left(\mathcal{T}_{p}\right)$ of vertices corresponds bijectively to the set of homothety classes of $\mathbb{Z}_{p}$-lattices of rank 2 in $\mathbb{Q}_{p}^{2}$. Two vertices $v$ and $v^{\prime}$ are said to be adjacent if they can be represented by lattices $L$ and $L^{\prime}$ such that the finite quotient $L / L^{\prime}$ has order $p$. If $v$ and $v^{\prime}$ are adjacent, the ordered pair $e=\left(v, v^{\prime}\right)$ is called an oriented edge, with origin $s(e)=v$ and target $t(e)=v^{\prime}$. Write $\mathcal{E}\left(\mathcal{T}_{p}\right)$ for the set of oriented edges of $\mathcal{T}_{p}$. Given $e=\left(v, v^{\prime}\right)$, let $\bar{e}=\left(v^{\prime}, v\right)$ be the opposite edge. Note that $\mathbf{P G L}_{2}\left(\mathbb{Q}_{p}\right)$ acts naturally on the left on $\mathcal{T}_{p}$, as a group of isometries. Let $v^{o}$ be the vertex corresponding to standard lattice $\mathbb{Z}_{p}^{2}$. Given $g \in \mathbf{P G L}_{2}\left(\mathbb{Q}_{p}\right)$, the assignment $g \mapsto g v^{o}$ induces an identification

$$
\mathbf{P G L}_{2}\left(\mathbb{Q}_{p}\right) / \mathbf{P G L}_{2}\left(\mathbb{Z}_{p}\right) \stackrel{=}{\longrightarrow} \mathcal{V}\left(\mathcal{T}_{p}\right) \text {. }
$$

Likewise, let $e^{o}$ be the oriented edge such that $s\left(e^{o}\right)=v^{o}$ and the stabilizer of $e^{o}$ in $\mathbf{P G L}\left(\mathbb{Q}_{p}\right)$ is equal to the group $\Gamma_{0}\left(p \mathbb{Z}_{p}\right)$ of matrices in $\mathbf{P G L}_{2}\left(\mathbb{Z}_{p}\right)$ which are upper triangular modulo $p$. The assignment $g \mapsto g e^{o}$ induces an identification

$$
\operatorname{PGL}_{2}\left(\mathbb{Q}_{p}\right) / \Gamma_{0}\left(p \mathbb{Z}_{p}\right) \stackrel{=}{\longrightarrow} \mathcal{E}\left(\mathcal{T}_{p}\right)
$$

Let

$$
\text { red }: \mathbb{P}_{1}\left(\mathbb{C}_{p}\right) \rightarrow \mathbb{P}_{1}\left(\overline{\mathbb{F}}_{p}\right)
$$


be the reduction map modulo the maximal ideal of the ring of integers of $\mathbb{C}_{p}$. Define a subset $A_{v^{o}}$ of $\mathcal{H}_{p}$ by

$$
A_{v^{\circ}}:=\left\{z \in \mathbb{P}_{1}\left(\mathbb{C}_{p}\right): \operatorname{red}(z) \notin \mathbb{P}_{1}\left(\mathbb{F}_{p}\right)\right\}
$$

More generally, given any vertex $v$, choose $g \in \mathbf{P G L}_{2}\left(\mathbb{Q}_{p}\right)$ such that $v=g v^{o}$, and let $A_{v}:=g A_{v^{o}}$. (Note that $A_{v}$ does not depend on the choice of $g$.) The sets $A_{v}$, obtained by excising $p+1$ disjoint open disks from $\mathbb{P}_{1}\left(\mathbb{C}_{p}\right)$, are examples of so-called connected affinoid domains in $\mathcal{H}_{p}$. Furthermore, define

$$
W_{] e^{o}[}:=\left\{z \in \mathbb{P}_{1}\left(\mathbb{C}_{p}\right): 1<|z|_{p}<p\right\} \subset \mathcal{H}_{p} .
$$

For any $e \in \mathcal{E}\left(\mathcal{T}_{p}\right)$, written as $e=g e^{o}$ for $g \in \mathbf{P G L}_{2}\left(\mathbb{Q}_{p}\right)$, set $W_{] e[}:=g W_{] e^{o}[}$. The set $W_{\text {]e }}$ is called the oriented wide open annulus attached to $e$, the orientation corresponding to the choice of the disk

$$
D_{e}:=\left\{z:\left|g^{-1} z\right|_{p} \geq p\right\} \subset \mathbb{P}_{1}\left(\mathbb{C}_{p}\right)
$$

in the complement of $W_{] e[}$. Finally, if $e=\left(v, v^{\prime}\right)$, define the standard affinoid subset attached to $e$ to be

$$
A_{[e]}:=A_{v} \cup W_{] e[} \cup A_{v^{\prime}} .
$$

The sets $A_{[e]}$ give a covering of $\mathcal{H}_{p}$ by standard affinoid subdomains whose intersection relations are reflected in the incidence relations on the tree. This allows us to define a reduction map

$$
r: \mathcal{H}_{p} \rightarrow \mathcal{V}\left(\mathcal{T}_{p}\right) \cup \mathcal{E}\left(\mathcal{T}_{p}\right)
$$

by sending $z$ to $v$ (resp. to $e$ ) if $z$ belongs to $A_{v}$ (resp. to $W_{] e[}$ ).

Definition 2.1 A function $f: \mathcal{H}_{p} \rightarrow \mathbb{C}_{p}$ is called rigid analytic if its restriction to each affinoid subset $A_{[e]}$ is a uniform limit (with respect to the sup norm) of a sequence of rational functions having poles outside $A_{[e]}$.

Fixing an embedding $\iota$ of $B$ into $M_{2}\left(\mathbb{Q}_{p}\right)$ yields a natural action on $\mathcal{H}_{p}$ of the group

$$
\Gamma:=\iota\left(R_{1}^{\times}\right) \subset \mathrm{SL}_{2}\left(\mathbb{Q}_{p}\right),
$$

where $R_{1}^{\times}$denotes the group of norm 1 elements in $R^{\times}$. The rigid analytic structure of $\mathcal{H}_{p}$ yields a rigid analytic structure on the quotient

$$
X_{\Gamma}:=\mathcal{H}_{p} / \Gamma
$$


which, by a $p$-adic variant of the Riemann existence theorem (or the GAGA principle) defines a curve over $\mathbb{C}_{p}$. This curve can be defined over $\mathbb{Q}_{p}$ and is equipped with a ring of Hecke correspondences $T_{n}$, arising from the fact that $\Gamma$ is arithmetic, whose definition is given in [JoLi2].

Furthermore, the Cerednik-Drinfeld theorem as formulated in [JoLi1], states that $X_{\Gamma}$ (just like the Riemann surface $\mathcal{H} / \Gamma_{0}(N)$ discussed in section 1 ) admits a model over $\mathbb{Q}$. This model is described as the Shimura curve attached to an Eichler $\mathbb{Z}$-order of level $N^{+}$in the indefinite quaternion algebra of discriminant $N^{-} p$, and is isomorphic to $X_{\Gamma}$ over the quadratic unramified extension of $\mathbb{Q}_{p}$. (For more background on Shimura curves, the reader may profitably consult the articles by Gross and Zhang in this volume. The article of [BoCa] gives a detailed account of Drinfeld's approach to the proof of the Cerednik-Drinfeld theorem.)

When combined with the Jacquet-Langlands correspondence (see for example section 3.5 of [BD3]), this result shows that the classical modular form

$$
f_{E}=\sum_{n \geq 1} a_{n} e^{2 \pi i n \tau}
$$

attached to $E$ as in section 1 corresponds to a weight 2 rigid analytic modular form on $\mathcal{H}_{p}$ with respect to $\Gamma$. More precisely, writing $T_{n}$ for the $n$-th Hecke correspondence on $X_{\Gamma}$, one has the following result which follows by combining work of Cerednik-Drinfeld and Jacquet-Langlands:

Theorem 2.2 There is a rigid analytic function

$$
f: \mathcal{H}_{p} \longrightarrow \mathbb{C}_{p}
$$

well-defined up to multiplication by elements of $\mathbb{C}_{p}^{\times}$, such that:

$$
\begin{aligned}
& \text { 1. } f(\gamma z)=(c z+d)^{2} f(z) \text { for all } \gamma=\left(\begin{array}{ll}
a & b \\
c & d
\end{array}\right) \in \Gamma \text {, } \\
& \text { 2. } T_{n} f=a_{n} f \text { for all } n \geq 1 \text { with }(n, N)=1 \text {. }
\end{aligned}
$$

The work of P. Schneider and J. Teitelbaum yields a concrete description, well-suited for numerical calculations, of the form $f$ in terms of its associated boundary measure.

Given a rigid analytic differential form $\omega=f(z) d z$ on $\mathcal{H}_{p}$, and an edge $e$ of $\mathcal{T}_{p}$, let $\left(f_{j}\right)$ be a sequence of rational functions on $\mathbb{C}_{p}$ converging uniformly 
to $f$ on $A_{[e]}$ and having poles outside $A_{[e]}$. The limit

$$
\lim _{j \rightarrow \infty} \sum_{t \in D_{e}} \operatorname{res}_{t}\left(f_{j}(z) d z\right)
$$

exists in $\mathbb{C}_{p}$ and depends only on $f$ and $e$, not on the approximating sequence $f_{j}$. It is called the $p$-adic annular residue of $f$ at $e$ and is denoted $\operatorname{res}_{e}(f(z) d z)$.

A function $\kappa$ on $\mathcal{E}\left(\mathcal{T}_{p}\right)$ with values in $\mathbb{C}_{p}$ is called a harmonic cocycle if

1. $\sum_{s(e)=v} \kappa(e)=0$ for all vertices $v$ (where the sum is taken over all the edges originating from $v$ ),

2. $\kappa(\bar{e})=-\kappa(e)$ for all oriented edges $e$.

Lemma 2.3 Let $f$ be a rigid analytic modular form of weight 2 on $\mathcal{H}_{p} / \Gamma$. The function

$$
\kappa_{f}: \mathcal{E}\left(\mathcal{T}_{p}\right) \longrightarrow \mathbb{C}_{p}, \quad e \mapsto \operatorname{res}_{e}(f(z) d z),
$$

is a $\mathbb{C}_{p}$-valued harmonic cocycle. Furthermore, $\kappa_{f}$ is $\Gamma$-invariant, that is, $\kappa_{f}(\gamma e)=\kappa_{f}(e)$.

Proof: The first statement follows directly from the residue theorem for rational differentials in light of the definition of the residue of $f$ at $e$. The $\Gamma$-invariance of $\kappa_{f}$ follows from the statement that

$$
\operatorname{res}_{\gamma e}(f(z) d z)=\operatorname{res}_{e}\left(f\left(\gamma^{-1} z\right) d\left(\gamma^{-1} z\right)\right),
$$

which is valid for all $\gamma \in \mathbf{S L}_{2}\left(\mathbb{Q}_{p}\right)$. See [Sch] for a more complete discussion which also applies to modular forms of higher weight.

Write $C_{\text {har }}^{\Gamma}$, respectively, $C_{\text {har }}(\mathbb{Q})^{\Gamma}$ for the space of $\Gamma$-invariant harmonic cocycles with values in $\mathbb{C}_{p}$, respectively, $\mathbb{Q}$. Note that the second space defines a $\mathbb{Q}$-structure on the first. Moreover, both spaces are equipped with an induced action of the Hecke algebra $\mathbb{T}_{\Gamma}$ attached to the curve $X_{\Gamma}$. It follows that the one-dimensional subspace of $C_{\mathrm{har}}^{\Gamma}$ on which $\mathbb{T}_{\Gamma}$ acts via the character defined by the form $f$ is generated by an element of $C_{\text {har }}(\mathbb{Q})^{\Gamma}$. Since $\mathcal{T}_{p} / \Gamma$ is a finite graph, an element $C_{\text {har }}^{\Gamma}$ is completely specified by its values on a finite set of orbit representatives. From now on, normalize the form $f$ by requiring that the set of values of $\kappa_{f}$ be contained in $\mathbb{Z}$ but in no proper ideal of $\mathbb{Z}$. This condition determines $f$ up to sign. 
Each ordered edge $e$ of $\mathcal{T}_{p}$ gives rise to a compact open subset $U_{e}$ of $\mathbb{P}_{1}\left(\mathbb{Q}_{p}\right)$ by the rule:

$$
U_{e}:=D_{e} \cap \mathbb{P}_{1}\left(\mathbb{Q}_{p}\right) .
$$

The cocycle $\kappa_{f}$ then gives rise to a $\Gamma$-invariant measure $\mu_{f}$ on $\mathbb{P}_{1}\left(\mathbb{Q}_{p}\right)$ satisfying the relation

$$
\int_{U_{e}} d \mu_{f}(x)=\kappa_{f}(e) .
$$

Theorem 2.4 (Teitelbaum) The form $f$ can be recovered from its boundary measure $\mu_{f}$ by the rule

$$
f(z)=\int_{\mathbb{P}_{1}\left(\mathbb{Q}_{p}\right)}\left(\frac{1}{z-t}\right) d \mu_{f}(t) .
$$

Sketch of proof. In view of the injectivity of the residue map, it is sufficient to check that the integral expression

$$
I(z):=\int_{\mathbb{P}_{1}\left(\mathbb{Q}_{p}\right)}\left(\frac{1}{z-t}\right) d \mu_{f}(t)
$$

is a rigid analytic modular form of weight 2 on $\mathcal{H}_{p} / \Gamma$ which has the same residues as $f$. To see that $I(z)$ is rigid analytic, note that the integral on the right in (11) is expressed as a limit of finite Riemann sums, each of which are rational functions having poles in $\mathbb{P}_{1}\left(\mathbb{Q}_{p}\right)$, and that these rational functions converge uniformly to $I(z)$ on any affinoid $A_{[e]}$. The definition of the $p$-adic annular residue given above makes it clear that

$$
\operatorname{res}_{e}(I(z) d z)=\int_{U_{e}} d \mu_{f}(t) .
$$

The $\Gamma$-invariance of $I(z) d z$ now follows from a direct calculation using the $\Gamma$-invariance of the measure $\mu_{f}$ combined with the fact that $\mu_{f}\left(\mathbb{P}_{1}\left(\mathbb{Q}_{p}\right)\right)=0$. See [Te], pp. 402-403 for a detailed proof and a more general statement which also applies to modular forms of higher weight.

Choose a branch

$$
\log _{p}: \mathbb{C}_{p}^{\times} \longrightarrow \mathbb{C}_{p}
$$

of the $p$-adic logarithm, and define the Coleman p-adic line integral associated to this choice by the rule

$$
\int_{\tau_{1}}^{\tau_{2}} f(z) d z:=\int_{\mathbb{P}_{1}\left(\mathbb{Q}_{p}\right)} \log _{p}\left(\frac{t-\tau_{2}}{t-\tau_{1}}\right) d \mu_{f}(t), \quad \tau_{1}, \tau_{2} \in \mathcal{H}_{p}
$$


Note that the integrand is a locally analytic $\mathbb{C}_{p}$-valued function on $\mathbb{P}_{1}\left(\mathbb{Q}_{p}\right)$ so that the integral converges in $\mathbb{C}_{p}$ (see [Te], p. 401). Equation (12) can be justified by the following formal calculation relying on theorem 2.4:

$$
\begin{aligned}
\int_{\tau_{1}}^{\tau_{2}} f(z) d z & =\int_{\tau_{1}}^{\tau_{2}} \int_{\mathbb{P}_{1}\left(\mathbb{Q}_{p}\right)}\left(\frac{1}{z-t}\right) d \mu_{f}(t) d z \\
& =\int_{\mathbb{P}_{1}\left(\mathbb{Q}_{p}\right)} \int_{\tau_{1}}^{\tau_{2}}\left(\frac{d z}{z-t}\right) d \mu_{f}(t) \\
& =\int_{\mathbb{P}_{1}\left(\mathbb{Q}_{p}\right)} \log _{p}\left(\frac{t-\tau_{2}}{t-\tau_{1}}\right) d \mu_{f}(t) .
\end{aligned}
$$

Because the $p$-adic measure $\mu_{f}$ comes from a harmonic cocycle taking values in $\mathbb{Z}$ and not just $\mathbb{Z}_{p}$, it is even possible to define the following multiplicative refinement of the Coleman line integral by formally exponentiating the expression in (12)

$$
\oint_{\tau_{1}}^{\tau_{2}} f(z) d z:=\oint_{\mathbb{P}_{1}\left(\mathbb{Q}_{p}\right)}\left(\frac{t-\tau_{2}}{t-\tau_{1}}\right) d \mu_{f}(t) \in \mathbb{C}_{p}^{\times},
$$

where

$$
\oint_{\mathbb{P}_{1}\left(\mathbb{Q}_{p}\right)} g(t) d \mu_{f}(t):=\lim _{\mathcal{C}=\left\{U_{\alpha}\right\}} \prod_{\alpha} g\left(t_{\alpha}\right)^{\mu_{f}\left(U_{\alpha}\right)}
$$

the limit is taken over increasingly fine covers $\mathcal{C}=\left\{U_{\alpha}\right\}$ of $\mathbb{P}_{1}\left(\mathbb{Q}_{p}\right)$ by disjoint compact open subsets, and $t_{\alpha} \in U_{\alpha}$ is any collection of sample points. This limit of "Riemann products" converges in $\mathbb{C}_{p}^{\times}$provided that the $\mathbb{C}_{p}^{\times}$-valued function $g(t)$ is locally analytic.

The multiplicative Coleman integral has the virtue over its more classical additive counterpart that it does not rely on a choice of $p$-adic logarithm, and carries more information. In fact, the two integrals are related by the formula

$$
\int_{\tau_{1}}^{\tau_{2}} f(z) d z=\log _{p}\left(\oint_{\tau_{1}}^{\tau_{2}} f(z) d z\right)
$$

The unramified upper half plane, denoted $\mathcal{H}_{p}^{\mathrm{nr}}$, is the set of $\tau \in \mathcal{H}_{p}$ whose image under the reduction map $r$ of equation (9) belongs to $\mathcal{V}\left(\mathcal{T}_{p}\right)$, i.e.,

$$
\mathcal{H}_{p}^{\mathrm{nr}}=\cup_{v \in \mathcal{V}\left(\mathcal{T}_{p}\right)} A_{v}
$$

Given two vertices $v_{1}$ and $v_{2}$ of $\mathcal{T}_{p}$, let $\sum_{e: v_{1} \rightarrow v_{2}}$ denote the sum taken over all the ordered edges $e$ in the path joining $v_{1}$ and $v_{2}$. We will have use for 
the following closed formula for the $p$-adic valuation of the multiplicative Coleman integral, which we content ourselves with stating in the special case where the $p$-adic endpoints of integration belong to $\mathcal{H}_{p}^{\mathrm{nr}}$.

Lemma 2.5 Let $\tau_{1}$ and $\tau_{2}$ be points in $\mathcal{H}_{p}^{\mathrm{nr}}$. Then

$$
\operatorname{ord}_{p}\left(\mathcal{f}_{\tau_{1}}^{\tau_{2}} f(z) d z\right)=\sum_{e: r\left(\tau_{1}\right) \rightarrow r\left(\tau_{2}\right)} \kappa_{f}(e) .
$$

Proof: Given $\tau_{1}, \tau_{2} \in \mathcal{H}_{p}^{\mathrm{nr}}$, and $t_{1}, t_{2} \in \mathbb{P}_{1}\left(\mathbb{Q}_{p}\right)$, let

$$
\left(\begin{array}{cc}
t_{1}: & \tau_{1} \\
t_{2}: & \tau_{2}
\end{array}\right)=\frac{\left(t_{2}-\tau_{2}\right)\left(t_{1}-\tau_{1}\right)}{\left(t_{2}-\tau_{1}\right)\left(t_{1}-\tau_{2}\right)}
$$

denote the usual cross-ratio. It is useful to have a formula for the $p$-adic valuation of this cross-ratio in terms of the combinatorics of $\mathcal{T}_{p}$. An ordered path on $\mathcal{T}_{p}$ is a sequence $\left(e_{j}\right)$ (either finite or infinite) of ordered edges of $\mathcal{T}_{p}$ satisfying $t\left(e_{j}\right)=s\left(e_{j+1}\right)$ for all $j$ for which $e_{j}$ is defined. The natural intersection pairing on $\mathcal{E}\left(\mathcal{T}_{p}\right)$ defined by

$$
e_{1} \cdot e_{2}=\left\{\begin{aligned}
1 & \text { if } e_{1}=e_{2} \\
-1 & \text { if } e_{1}=\bar{e}_{2} \\
0 & \text { otherwise }
\end{aligned}\right.
$$

extends by $\mathbb{Z}$-linearity to the set of paths of $\mathcal{T}_{p}$, and $\gamma_{1} \cdot \gamma_{2}$ is finite provided that the paths $\gamma_{1}$ and $\gamma_{2}$ do not share a half line in common. Let $\left[v_{1} \rightarrow v_{2}\right]$ denote the finite path joining the vertices $v_{1}$ and $v_{2}$, and let $\left(t_{1} \rightarrow t_{2}\right)$ denote the geodesic joining the points $t_{1}$ and $t_{2}$ of $\mathbb{P}_{1}\left(\mathbb{Q}_{p}\right)$ viewed as ends of $\mathcal{T}_{p}$. We claim that

$$
\operatorname{ord}_{p}\left(\begin{array}{cc}
t_{1}: & \tau_{1} \\
t_{2}: & \tau_{2}
\end{array}\right)=\left(t_{1} \rightarrow t_{2}\right) \cdot\left[r\left(\tau_{1}\right) \rightarrow r\left(\tau_{2}\right)\right] .
$$

In light of the fact that the action of $\mathbf{P S L}_{2}\left(\mathbb{Q}_{p}\right)$ preserves both the cross-ratio and the inner product on paths, and acts 3-point transitively on $\mathbb{P}_{1}\left(\mathbb{Q}_{p}\right)$, it is enough to verify this formula when $t_{1}=0$ and $t_{2}=\infty$. Let $v_{j}$ (with $j \in \mathbb{Z})$ denote the vertices on $(0 \rightarrow \infty)$, numbered consecutively in such a way that $v_{0}=v^{o}$ and that the end $\left(v_{0}, v_{1}, v_{2}, \ldots,\right)$ corresponds to 0 while the end $\left(v_{0}, v_{-1}, v_{-2}, \ldots\right)$ corresponds to $\infty$. Once this is done, let $\mathcal{T}^{j}$ denote the largest connected subtree of $\mathcal{T}_{p}$ containing $v_{j}$ and no edge on $(0 \rightarrow \infty)$. It follows directly from the definition of the reduction map, that $r(\tau)$ belongs 
to $\mathcal{T}^{0}$ if and only if $\operatorname{ord}_{p}(\tau)=0$. Since the Möbius transformation $\tau \mapsto p \tau$ preserves the geodesic $(0 \rightarrow \infty)$, sending $v_{j}$ to $v_{j+1}$ for all $j \in \mathbb{Z}$, it follows that

$$
r(\tau) \in \mathcal{T}^{j} \quad \text { if and only if } \quad \operatorname{ord}_{p}(\tau)=j .
$$

Suppose that $\tau_{1}$ and $\tau_{2}$ map to vertices of $\mathcal{T}^{n_{1}}$ and $\mathcal{T}^{n_{2}}$ respectively under the reduction map. A direct calculation shows that

$$
\operatorname{ord}_{p}\left(\begin{array}{cc}
0: & \tau_{1} \\
\infty: & \tau_{2}
\end{array}\right)=\operatorname{ord}_{p}\left(\tau_{1} / \tau_{2}\right)=n_{1}-n_{2}
$$

while clearly

$$
(0 \rightarrow \infty) \cdot\left[r\left(\tau_{1}\right) \rightarrow r\left(\tau_{2}\right)\right]=n_{1}-n_{2}
$$

Formula (16) follows from this.

By the additivity of both members of the equality (15) to be proven, it may now be assumed that $\tau_{1}$ and $\tau_{2}$ reduce to adjacent vertices, so that the path joining them consists of a single oriented edge $e:=\left(r\left(\tau_{1}\right), r\left(\tau_{2}\right)\right)$. Equation (13) yields

$$
\operatorname{ord}_{p}\left(\mho_{\tau_{1}}^{\tau_{2}} f(z) d z\right):=\int_{\mathbb{P}_{1}\left(\mathbb{Q}_{p}\right)} \operatorname{ord}_{p}\left(\frac{t-\tau_{2}}{t-\tau_{1}}\right) d \mu_{f}(t) .
$$

Let

$$
\phi(t):=\operatorname{ord}_{p}\left(\frac{t-\tau_{2}}{t-\tau_{1}}\right)
$$

be the expression appearing as the integrand in the right hand side of this equation. Setting $t_{1}=t$ and $t_{2}=\infty$ in equation (16) shows that

$$
\phi(t)=\left\{\begin{aligned}
+1 & \text { if } e \text { belongs to }(\infty \rightarrow t) \\
-1 & \text { if } \bar{e} \text { belongs to }(\infty \rightarrow t) \\
0 & \text { otherwise }
\end{aligned}\right.
$$

It follows that $\phi(t)$ is locally constant on the disjoint covering $\left\{U_{e}, U_{\bar{e}}\right\}$ of $\mathbb{P}_{1}\left(\mathbb{Q}_{p}\right)$. It may be assumed, possibly at the cost of interchanging $z_{1}$ and $z_{2}$, that $e$ (and not $\bar{e}$ ) belongs to the path $(\infty \rightarrow t)$ for some $t$. Then, one has $\phi(t)=1$ on $U_{e}$ and $\phi(t)=0$ on $U_{\bar{e}}$. Hence, by (17),

$$
\operatorname{ord}_{p} \bigcup_{\tau_{1}}^{\tau_{2}} f(z) d z=\int_{U_{e}} d \mu_{f}(t)=\kappa_{f}(e)
$$


as was to be shown. (For more on such formulae, see for example the arguments in the proof of lemma 5.6 of [Man2].)

The multiplicative Coleman integral yields a rigid analytic uniformisation of $E$ by the curve $X_{\Gamma}$ defined in equation (10). Extending by linearity, the formula (13) gives rise to a map from the degree zero divisors on $\mathcal{H}_{p}$ to $\mathbb{C}_{p}^{\times}$. This map descends to a map from $\operatorname{Pic}^{0}\left(X_{\Gamma}\right)$ to $\mathbb{C}_{p}^{\times} / q^{\mathbb{Z}}$,

$$
\left(\tau_{2}\right)-\left(\tau_{1}\right) \mapsto \oint_{\left(\tau_{2}\right)-\left(\tau_{1}\right)} f(z) d z:=\oint_{\tau_{1}}^{\tau_{2}} f(z) d z
$$

where $\operatorname{Pic}^{0}\left(X_{\Gamma}\right)$ denotes the jacobian of $X_{\Gamma}$, and where the $p$-adic period $q$ is an element of $p \mathbb{Z}_{p}$. The quotient $\mathbb{C}_{p}^{\times} / q^{\mathbb{Z}}$ defines an elliptic curve over $\mathbb{C}_{p}$ isogenous to $E$.

From now on, assume (possibly after replacing $E$ by a curve which is $\mathbb{Q}$-isogenous to it) that the Tate $p$-adic uniformisation

$$
\eta_{p}: \mathbb{C}_{p}^{\times} \longrightarrow E\left(\mathbb{C}_{p}\right)
$$

induces an isomorphism from $\mathbb{C}_{p}^{\times} / q^{\mathbb{Z}}$ onto $E\left(\mathbb{C}_{p}\right)$. Choose a correspondence $\theta \in \mathbb{T}$ which maps $\operatorname{Div}\left(X_{\Gamma}\right)$ to $\operatorname{Div}^{0}\left(X_{\Gamma}\right)$ (such as $T_{\ell}-(\ell+1)$ for a prime $\ell$ not dividing $N)$. Replace the multiplicative integral of (19) by the new expression

$$
\oiint_{\tau_{1}}^{\tau_{2}} f(z) d z:=\Varangle_{\theta\left(\left(\tau_{2}\right)-\left(\tau_{1}\right)\right)} f(z) d z .
$$

The redefined integral of $(21)$ extends to the whole Picard variety $\operatorname{Pic}\left(X_{\Gamma}\right)$ by the rule

$$
(\tau) \mapsto \rtimes^{\tau} f(z) d z:=\oint_{\theta \tau} f(z) d z .
$$

The map of (22) should be viewed as the definition of the concept of a semiindefinite (multiplicative) integral in the current context. Note the relation in $\mathbb{C}_{p}^{\times} / q^{\mathbb{Z}}$ :

$$
\left(\Varangle^{\tau_{2}} f(z) d z\right) \div\left(\chi^{\tau_{1}} f(z) d z\right)=\Varangle_{\tau_{1}}^{\tau_{2}} f(z) d z .
$$

The semi-indefinite integral can also be described in terms of values of $p$-adic theta functions (so that the definite integral is a ratio of such values). 
The next result makes explicit the relation between the additive Coleman integral corresponding to a choice of $\operatorname{logarithm} \log _{p}$ and the harmonic cocycle $\kappa_{f}$. Define the $\mathcal{L}$-invariant attached to $f$ by the rule

$$
\mathcal{L}_{f}:=\frac{\log _{p}(q)}{\operatorname{ord}_{p}(q)} .
$$

Proposition 2.6 Let $z$ be any point in $\mathcal{H}_{p}$ and let $v$ be any vertex of $\mathcal{T}_{p}$. For all $\gamma \in \Gamma$, the equality

$$
\int_{z}^{\gamma z} f(\tau) d \tau=\mathcal{L}_{f} \cdot \sum_{e: v \rightarrow \gamma v} \kappa_{f}(e)
$$

holds.

Proof: Note that the left-hand side of equation (23) does not depend on the choice of $z$, as a consequence of the $\Gamma$-invariance of the differential $f(\tau) d \tau$. In particular, $z$ can be taken in $\mathcal{H}_{p}^{\text {nr }}$. Similarly, the right-hand side of equation (23) does not depend on the choice of $v$. The theory of $p$-adic uniformisation recalled above yields

$$
\oiint_{z}^{\gamma z} f(\tau) d \tau=q^{n_{\gamma}}
$$

for all $\gamma$, where $n_{\gamma}$ is an integer. Taking $\log _{p}$ and $\operatorname{ord}_{p}$ of (24), and comparing the resulting equalities, gives

$$
\int_{z}^{\gamma z} f(\tau) d \tau=\mathcal{L}_{f} \cdot \operatorname{ord}_{p} \oint_{z}^{\gamma z} f(\tau) d \tau .
$$

Proposition 2.6 follows by combining (25) with lemma 2.5.

\section{The period integral}

Let $K / \mathbb{Q}$ be a quadratic algebra contained in $\mathbb{C}_{p}$, and assume that it satisfies the following modified Heegner hypothesis:

1. $K$ is an imaginary quadratic field;

2. all the primes dividing $N^{-}$are inert in $K$;

3. all the primes dividing $N^{+}$are split in $K$; 
4. $p$ is inert in $K$.

Conditions 1 and 2 are precisely what is needed to ensure the existence of an embedding of $K$ into $B$, the definite quaternion algebra of discriminant $N^{-}$ introduced at the start of this section.

Let $\mathcal{O}$ be any $\mathbb{Z}[1 / p]$-order of $K$ of conductor prime to $N$. (So that $\mathcal{O}$ is a subring of $K$ which is free of rank two over $\mathbb{Z}[1 / p]$.) Choose an Eichler $\mathbb{Z}[1 / p]$-order $R$ of level $N^{+}$in $B$. An orientation on the $\operatorname{ring} T=\mathcal{O}$ or $T=R$ is a surjective ring homomorphism

$$
\mathfrak{o}: T \longrightarrow\left(\mathbb{Z} / N^{+} \mathbb{Z}\right) \times \prod_{\ell \mid N^{-}} \mathbb{F}_{\ell^{2}}
$$

Note that such an orientation exists on $\mathcal{O}$ thanks to conditions 2 and 3 satisfied by $K$.

Fix such orientations on $\mathcal{O}$ and on $R$ once and for all. Let $\Psi: K \longrightarrow B$ be an embedding of algebras. By condition 4 satisfied by $K$, the group $\iota \Psi\left(K^{\times}\right)$ acting on $\mathcal{T}_{p}$ has precisely one fixed vertex $v_{\Psi}$. An embedding $\Psi: K \longrightarrow B$ is said to be an oriented optimal embedding of $\mathcal{O}$ into $R$ (relative to the fixed orientations on $\mathcal{O}$ and $R$ ) if:

1. $\Psi$ embeds $\mathcal{O}$ optimally as a subring of $R$;

2. The map $\Psi$ is compatible with the orientations on $\mathcal{O}$ and $R$ in the obvious sense;

3. The map $\Psi$ is oriented at $p$, in the sense that

$$
v_{\Psi} \text { belongs to } \mathbf{S L}_{2}\left(\mathbb{Q}_{p}\right) v^{o} \text {. }
$$

The conditions imposed on $K$ above ensure the existence of oriented optimal embeddings. It can be verified, just as in section 1 , that the group $\Gamma=\iota\left(R_{1}^{\times}\right)$ acts on them by conjugation; as in that section, let $\operatorname{Emb}(\mathcal{O}, R)$ denote the set of $\Gamma$-conjugacy classes of oriented optimal embeddings of $\mathcal{O}$ into $R$. The period integral considered here is attached to the class of an embedding $\Psi$ in $\operatorname{Emb}(\mathcal{O}, R)$.

Write $K_{p}$ for the local field $K \otimes \mathbb{Q}_{p}$. The torus $K_{p}^{\times}$acting on $\mathcal{H}_{p}$ via the embedding $\iota \Psi$ has a unique fixed point $\tau_{\Psi}$ satisfying

$$
\iota \Psi(\alpha)\left(\begin{array}{c}
\tau_{\Psi} \\
1
\end{array}\right)=\alpha\left(\begin{array}{c}
\tau_{\Psi} \\
1
\end{array}\right), \quad \text { for all } \alpha \in K_{p}^{\times} .
$$


This fixed point belongs to $\mathcal{H}_{p} \cap K_{p}$; let $\bar{\tau}_{\Psi}$ denote its conjugate under the action of $\operatorname{Gal}\left(K_{p} / \mathbb{Q}_{p}\right)$.

Definition 2.7 The period integrals associated to the embedding $\Psi$ are defined to be

$$
I_{\Psi}:=\oint_{\bar{\tau}_{\Psi}}^{\tau_{\Psi}} f(z) d z \in \mathbb{C}_{p}^{\times} \quad \text { and } \quad J_{\Psi}:=\oint^{\tau_{\Psi}} f(z) d z \in \mathbb{C}_{p}^{\times} / q^{\mathbb{Z}} .
$$

The next lemma follows directly from formula (13).

Lemma 2.8 The periods $I_{\Psi}$ and $J_{\Psi}$ belong to $K_{p}^{\times}$and $K_{p}^{\times} / q^{\mathbb{Z}}$, respectively.

Note that $J_{\Psi}$ and $I_{\Psi}$ are related by the equality

$$
J_{\Psi} / \bar{J}_{\Psi}=I_{\Psi} \quad\left(\bmod q^{\mathbb{Z}}\right) .
$$

The following theorem states that the images under $\eta_{p}$ of the period integrals $J_{\Psi}$ and $I_{\Psi}$ define global points on the elliptic curve $E$. Let $w=1$ if $E / \mathbb{Q}_{p}$ has split multiplicative reduction, and $w=-1$ otherwise. Let $H$ be the ring class field of $K$ attached to the order $\mathcal{O}$. Note that $p$ is totally split in the extension $H / K$. Fix an embedding of $H$ into $K_{p} \subset \mathbb{C}_{p}$. This amounts to choosing a prime $\mathfrak{p}$ of $H$ above $p$. In particular $E(H)$ becomes a subgroup of $E\left(\mathbb{C}_{p}\right)$ thanks to this choice. Write $\sigma_{\mathfrak{p}}$ for the Frobenius element of $\mathfrak{p}$ in $\operatorname{Gal}(H / \mathbb{Q})$.

Theorem 2.9 ([BD2]) For each optimal embedding $\Psi$ of $\mathcal{O}$ into $R$, the point $\eta_{p}\left(J_{\Psi}\right)$ is a global point $P_{\Psi}$ in $E(H)$. In particular $\eta_{p}\left(I_{\Psi}\right)$ is equal to the global point $P_{\Psi}-w \sigma_{\mathfrak{p}} P_{\Psi}$. Furthermore, for all $\mathfrak{a} \in \operatorname{Pic}(\mathcal{O})$,

$$
\eta_{p}\left(J_{\Psi^{\mathfrak{a}}}\right)=\operatorname{rec}(\mathfrak{a})^{-1} \cdot \eta_{p}\left(J_{\Psi}\right) .
$$

The proof of this theorem follows from Drinfeld's moduli interpretation of the $p$-adic upper half plane $\mathcal{H}_{p}$. This interpretation implies that the point on the Shimura curve model of $X_{\Gamma}$ which corresponds to $\tau_{\Psi}$ is a Heegner point defined over $H$. See chapter 5 of [BD2] for details.

Remarks:

1. Note that the Shimura reciprocity law described above for the periods $J_{\Psi}$ does not extend in the natural way to the periods $I_{\Psi}$, because the substitution $\tau_{\Psi} \mapsto \bar{\tau}_{\Psi}$ does not commute with the action of $\operatorname{Pic}(\mathcal{O})$ on the optimal embeddings $\Psi$. More precisely, one has

$$
\bar{\Psi}^{\mathfrak{a}^{-1}}=\overline{\Psi^{\mathfrak{a}}},
$$


so that by the Shimura reciprocity law above

$$
\eta_{p}\left(I_{\Psi^{a}}\right)=\eta_{p}\left(J_{\Psi^{a}} \div J_{\bar{\Psi}^{a}-1}\right) .
$$

Thus the expression of the right is not equal in general to $\operatorname{rec}(\mathfrak{a})^{-1} \eta_{p}\left(I_{\Psi}\right)$ unless $\mathfrak{a}=\mathfrak{a}^{-1}$ in $\operatorname{Pic}(\mathcal{O})$.

2. (The relation with $p$-adic L-functions.) The periods $I_{\Psi}$ are notable for their simple relation with special values of certain anticyclotomic $p$-adic $L$ functions. More precisely, let $\log _{p}$ denote the standard $p$-adic logarithm

$$
\log _{p}: K_{p, 1}^{\times} \rightarrow K_{p},
$$

where $K_{p, 1}^{\times}$is the compact group of norm 1 elements in $K_{p}^{\times}$. In [BDIS], building on an idea of Schneider, a partial anticyclotomic $p$-adic $L$-function is attached to $f$ and the embedding $\Psi$ by taking a $p$-adic Mellin transform of the measure attached to the form $f$ :

$$
L_{p}(f, \Psi, s)=\int_{\mathbb{P}_{1}\left(\mathbb{Q}_{p}\right)}\left(\frac{t-\tau_{\Psi}}{t-\bar{\tau}_{\Psi}}\right)^{s-1} d \mu_{f}(t) .
$$

Note that the expression $\left(\frac{t-\tau_{\Psi}}{t-\bar{\tau}_{\Psi}}\right)$ appearing in the integrand belongs to $K_{p, 1}^{\times}$, so that the $p$-adic exponentiation that occurs there can be defined by

$$
\alpha^{s}:=\exp \left(s \log _{p}(\alpha)\right),
$$

a definition which is independent of a choice of logarithm.

Equation (27) defining the $p$-adic $L$-function can be justified by noting that the measure $\mu_{f}$ satisfies the following interpolation formula with respect to classical special values, which for the sake of illustration we give only in the case where $\operatorname{Pic}(\mathcal{O})$ has cardinality 1 so that there is a unique optimal embedding $\Psi$ of $\mathcal{O}$ into $R$ up to conjugation by $\Gamma$. If $\chi: K_{p, 1}^{\times} \longrightarrow \mathbb{C}_{p}^{\times}$is a finite order character, viewed as a (complex or $p$-adic) character of $\operatorname{Gal}(\bar{K} / K)$ in the usual way, then

$$
\left|\int_{\mathbb{P}_{1}\left(\mathbb{Q}_{p}\right)} \chi\left(\frac{t-\tau_{\Psi}}{t-\bar{\tau}_{\Psi}}\right) d \mu_{f}(t)\right|^{2} \doteq L(E / K, \chi, 1),
$$

where the symbol $\doteq$ denotes equality up to a simple fudge factor. See for example $[\mathrm{Zh}]$ where this formula is proved. 
It is apparent from the definition of the $p$-adic $L$-function given in (27) that

$$
L_{p}^{\prime}(f, \Psi, 1)=\log _{p}\left(I_{\Psi}\right) .
$$

Combined with theorem 2.9, which expresses $\eta_{p}\left(I_{\Psi}\right)$ in terms of Heegner points, equation (28) can be viewed as a rigid analytic variant of the GrossZagier formula. Recall that Zhang's generalisation of the Gross-Zagier theorem [Zh] relates the Néron-Tate height of the points $P_{\Psi}$ to the derivative of certain complex Rankin $L$-series attached to $f$ and to $K$.

It is interesting to note that while the period $I_{\Psi}$ admits a natural interpretation in terms of the first derivative of an (anticyclotomic) $p$-adic $L$-function, a similar interpretation is not known for the periods $J_{\Psi}$ even though these periods are more natural insofar as they satisfy a cleanly stated Shimura reciprocity law. A clue for understanding this phenomenon is provided by the computations performed in [BD1], which relate $I_{\Psi}$ to a (partial) $p$-adic regulator expressing the leading term of the partial $p$-adic $L$-function $L_{p}(f, \Psi, s)$. Following the ideas of $[\mathrm{MTT}]$, this regulator is defined on the extended Mordell-Weil group of $E$, by pairing $P_{\Psi}$ with the Tate period $q$.

\section{Forms on $\mathcal{T}_{p} \times \mathcal{H}$}

One of the questions which motivates this article is the apparent difficulty of extending the theory of complex multiplication (and hence, the Heegner point construction) to the setting where $K$ is a real quadratic field. The difficulty (at least on a superficial level) can be traced to two fundamentally different causes in the settings that were covered in the previous two sections.

1. In section 1 , the difficulty comes about from the fact that the real quadratic subalgebras of $M_{2}(\mathbb{Q})$ do not have fixed points on $\mathcal{H}^{*}$, so that the period integral $(7)$ cannot be extended to this setting in any obvious way.

2. In section 2 , the difficulty can be ascribed to the fact that the discrete subgroups of $\mathbf{S L}_{2}\left(\mathbb{Q}_{p}\right)$ occuring in the Cerednik-Drinfeld theory come from unit groups of $\mathbb{Z}[1 / p]$-orders of definite quaternion algebras; but such quaternion algebras contain no real quadratic subalgebras. 
If $B$ is an indefinite quaternion algebra over $\mathbb{Q}$ for which the prime $p$ is split, and $R$ a $\mathbb{Z}[1 / p]$-order in $B$, one can of course always consider the group

$$
\Gamma:=\iota\left(R_{1}^{\times}\right),
$$

but this group does not act discretely on $\mathcal{H}_{p}$ or on $\mathcal{H}$. Rather, it acts discretely on the product $\mathcal{H}_{p} \times \mathcal{H}$.

It is from this point of view that a theory of mixed $p$-adic and archimedean modular forms becomes germane to the concerns of this article. Ihara [I] and Stark [St] studied this question in their papers on mixed place modular forms, and indeed the classical theory of complex multiplication plays an important role in the work of Thara. The problem of defining a sensible notion of analytic function on $\mathcal{H}_{p} \times \mathcal{H}$ seems inextricably linked to that of finding a well-behaved tensor product of $\mathbb{C}_{p}$ with $\mathbb{C}$, which appears to be a difficult task. However, there is a good compromise, at least in the special case where $B$ is the split quaternion algebra $M_{2}(\mathbb{Q})$.

Assume for this section that the conductor $N$ of $E$ is of the form $p M$ with $(p, M)=1$. To fix ideas, define $\Gamma$ as in the introduction:

$$
\Gamma:=\left\{\left(\begin{array}{ll}
a & b \\
c & d
\end{array}\right) \in \mathbf{S L}_{2}(\mathbb{Z}[1 / p]) \quad \text { such that } M \text { divides } c\right\} .
$$

In other words, if $R=\mathrm{M}_{0}(M) \otimes \mathbb{Z}[1 / p]$ denotes the standard Eichler $\mathbb{Z}[1 / p]$ order of level $M$ in $\mathrm{M}_{2}(\mathbb{Q})$, consisting of the matrices with entries in $\mathbb{Z}[1 / p]$ which are upper triangular modulo $M$, then $\Gamma=R_{1}^{\times}$. The group $\Gamma \subset \mathbf{S L}_{2}(\mathbb{Q})$ acts by linear fractional transformations both on $\mathcal{H}_{p}$ and on $\mathcal{H}$, yielding a discrete discontinuous action on the product $\mathcal{H}_{p} \times \mathcal{H}$. Moreover, $\Gamma$ acts transitively on the set of unordered edges of $\mathcal{T}_{p}$, and has exactly two orbits on $\mathcal{E}\left(\mathcal{T}_{p}\right)$, represented by $e^{o}$ and $\bar{e}^{o}$ respectively. Recall that $v^{o}$ is the base vertex of $\mathcal{T}_{p}$, characterized by the property that its stabiliser $\Gamma_{v^{o}}$ in $\Gamma$ is equal to $\Gamma_{0}(M)$, and that $e^{o}$ is the edge characterized by the properties $\operatorname{source}\left(e^{o}\right)=v^{o}$ and $\Gamma_{e^{o}}=\Gamma_{0}(N)$.

In theorem 2.4 of section 2 we saw that rigid analytic modular forms on $\mathcal{H}_{p} / \Gamma$ are naturally associated, and completely determined by, certain $\mathbb{C}_{p}$-valued functions $\mathcal{E}\left(\mathcal{T}_{p}\right)$ which are $\Gamma$-invariant and satisfy the harmonic cocycle property:

$$
\begin{gathered}
\sum_{s(e)=v} \kappa(e)=0 \text { for all } v \in \mathcal{V}\left(\mathcal{T}_{p}\right), \\
\text { and } \kappa(\bar{e})=-\kappa(e) \text { for all } e \in \mathcal{E}\left(\mathcal{T}_{p}\right) .
\end{gathered}
$$


Thus one might surmise that the role of this associated harmonic cocycle, for an "invariant differential 2-form on $\left(\mathcal{H}_{p} \times \mathcal{H}\right) / \Gamma$ ", should be played by a function $\kappa$ on $\mathcal{E}\left(\mathcal{T}_{p}\right)$ with values in a space of complex analytic functions on $\mathcal{H}$ satisfying the obvious harmonicity and $\Gamma$-invariance properties.

This brings us to the key definition of this section.

Definition 3.1 A cusp form of weight 2 on $\left(\mathcal{H}_{p} \times \mathcal{H}\right) / \Gamma$ is a function

$$
f: \mathcal{E}\left(\mathcal{T}_{p}\right) \times \mathcal{H} \rightarrow \mathbb{C}
$$

satisfying the following properties.

1. (Analyticity) For every edge e of $\mathcal{T}_{p}, f_{e}(z):=f(e, z)$ is a holomorphic function such that $f_{\bar{e}}=-f_{e}$, and

$$
\sum_{s(e)=v} f_{e}=0, \text { for every vertex } v \in \mathcal{V}\left(\mathcal{T}_{p}\right)
$$

2. (Г-invariance) For all $\gamma \in \Gamma, f(\gamma e, \gamma z) d(\gamma z)=f(e, z) d z$.

3. (Cuspidality) For every edge e, the function $f_{e}$ is a complex cusp form of weight 2 on $\Gamma_{e}$, the stabiliser of $e$ in $\Gamma$.

Let $S_{2}\left(\left(\mathcal{H}_{p} \times \mathcal{H}\right) / \Gamma\right)$ denote the space of cusp form of weight 2 on $\left(\mathcal{H}_{p} \times \mathcal{H}\right) / \Gamma$. As usual, write $S_{2}\left(\mathcal{H} / \Gamma_{0}(N)\right)$ for the space of weight 2 cusp forms on $\Gamma_{0}(N)$. Note that one has two natural degeneracy maps

$$
S_{2}\left(\mathcal{H} / \Gamma_{0}(N)\right) \rightrightarrows S_{2}\left(\mathcal{H} / \Gamma_{0}(M)\right)
$$

The forms killed by both degeneracy maps are called new at $p$. Denote the subspace of forms that are new at $p$ by $S_{2}^{p-\text { new }}\left(\mathcal{H} / \Gamma_{0}(N)\right)$. The proposition below shows that $S_{2}\left(\left(\mathcal{H}_{p} \times \mathcal{H}\right) / \Gamma\right)$ is a familiar object, identified directly with the $p$-new subspace of $S_{2}\left(\mathcal{H} / \Gamma_{0}(N)\right)$.

Proposition 3.2 The map $f \mapsto f_{e^{o}}$ defines an isomorphism

$$
S_{2}\left(\left(\mathcal{H}_{p} \times \mathcal{H}\right) / \Gamma\right) \stackrel{\sim}{\rightarrow} S_{2}^{p-\text { new }}\left(\mathcal{H} / \Gamma_{0}(N)\right) .
$$

Proof. Since the stabiliser $\Gamma_{e^{o}}$ of $e$ in $\Gamma$ is equal to $\Gamma_{0}(N)$, it follows from part 3 of definition 3.1 that $f_{e^{o}}$ belongs to $S_{2}\left(\mathcal{H} / \Gamma_{0}(N)\right)$, so that the assignment $f \mapsto f_{e^{o}}$ defines a linear map $S_{2}\left(\left(\mathcal{H}_{p} \times \mathcal{H}\right) / \Gamma\right) \rightarrow S_{2}\left(\mathcal{H} / \Gamma_{0}(N)\right)$. To see that 
this assignment is injective, note that if $f_{e^{o}}$ is 0 , then so is $f_{\bar{e}^{o}}=-f_{e^{o}}$, and hence

$$
f_{e}=0 \text { for all } e \in \mathcal{E}\left(\mathcal{T}_{p}\right)=\Gamma e^{o} \cup \Gamma \bar{e}^{o} .
$$

Next, we claim that the image of $S_{2}\left(\left(\mathcal{H}_{p} \times \mathcal{H}\right) / \Gamma\right)$ is precisely the space $S_{2}^{p-\text { new }}\left(\mathcal{H} / \Gamma_{0}(N)\right)$. Let $f_{0}$ be a modular form in this space, and define

$$
f_{e}(z) d z=f_{0}\left(\gamma^{-1} z\right) d\left(\gamma^{-1} z\right) \text { for } e=\gamma e^{o} \in \Gamma e^{0} .
$$

Extend this definition to $e \in \Gamma \bar{e}^{o}$ by setting $f_{e}=-f_{\bar{e}}$. It is easy to see that the collection $\left\{f_{e}\right\}$ satisfies all the properties of definition 3.1 with the possible exception of the harmonicity condition given in equation (30). To see that this latter condition is satisfied as well, note that by the definition of $p$-new-forms, $f_{0}$ satisfies

$$
\begin{gathered}
\sum_{\gamma \in \Gamma_{0}(N) / \Gamma_{0}(M)} f_{0}\left(\gamma^{-1} z\right) d\left(\gamma^{-1} z\right)=0 \\
\text { and } \sum_{\gamma \in \Gamma_{0}(N) / \Gamma_{0}(M)} f_{0}\left(\gamma^{-1} \alpha^{-1} z\right) d\left(\gamma^{-1} \alpha^{-1} z\right)=0,
\end{gathered}
$$

where $\alpha \in \mathrm{GL}_{2}^{+}(\mathbb{Z}[1 / p])$ is an element of the normaliser of $\Gamma_{0}(N)$ which does not belong to $\Gamma_{0}(N)$. Note that $\alpha e^{o}=\overline{e^{o}}$, so that $v^{1}:=\alpha v^{o}$ is the target of $e^{o}$. By strong approximation (which in this setting amounts to the Chinese Remainder theorem), the natural embedding of $\mathbf{S L}_{2}(\mathbb{Z})$ into $\mathbf{S L}_{2}\left(\mathbb{Z}_{p}\right)$ identifies the coset space $\Gamma_{0}(N) / \Gamma_{0}(M)$ with $\mathbf{S L}_{2}\left(\mathbb{Z}_{p}\right) / \Gamma_{0}\left(p \mathbb{Z}_{p}\right)$, so that

$$
\left\{\gamma e^{o}: \gamma \in \Gamma_{0}(N) / \Gamma_{0}(M)\right\}
$$

is a complete list of edges with source $v^{o}$, while

$$
\left\{\alpha \gamma e^{o}: \gamma \in \Gamma_{0}(N) / \Gamma_{0}(M)\right\}
$$

is a complete list of edges with edges with source $v^{1}$. Hence equations (31) and (32) just amount to the statement that $\left\{f_{e}\right\}$ is harmonic at $v^{o}$ and $v^{1}$ :

$$
\sum_{e: s(e)=v^{o}} f_{e}=0 ; \quad \sum_{e: s(e)=v^{1}} f_{e}=0
$$

Harmonicity at all other vertices now follows from the $\Gamma$-equivariance built into the definition of $f$ in terms of $f_{0}$, in light of the fact that $\mathcal{V}\left(\mathcal{T}_{p}\right)=$ 
$\Gamma v^{o} \cup \Gamma v^{1}$. Hence $f$ is a cusp form of weight 2 on $\left(\mathcal{H}_{p} \times \mathcal{H}\right) / \Gamma$, satisfying $f_{e^{o}}=f_{0}$, and therefore the image of the assigment $f \mapsto f_{e^{o}}$ contains the forms that are new at $p$. That these are the only forms in the image follows from the equivalence of equation (33) with equations (31) and (32).

Thanks to proposition 3.2, the space $S_{2}\left(\left(\mathcal{H}_{p} \times \mathcal{H}\right) / \Gamma\right)$ inherits an action of the Hecke operators $T_{n}$ (for $\left.\operatorname{gcd}(n, N)=1\right)$ from the familiar action of Hecke operators on $S_{2}^{p-\text { new }}\left(\mathcal{H} / \Gamma_{0}(N)\right)$. This Hecke action can be described directly as in formula (46) of [Da].

Given $\gamma \in \mathbf{P G L}_{2}\left(\mathbb{Q}_{p}\right)$, the quantity $|\gamma|_{p}:=\operatorname{ord}_{p}(\operatorname{det}(\gamma))$ is well-defined modulo 2. Let $w$ denote the negative of the eigenvalue of the Atkin-Lehner involution $W_{p}$ acting on $f_{0}$. Thus, $w$ is equal to 1 (resp. -1 ) if the abelian variety attached to $f_{0}$ has split (resp. non-split) multiplicative reduction over $\mathbb{Q}_{p}$. The following lemma describes an invariance property of $f$ under the action of the larger group $\iota\left(R_{+}^{\times}\right) \supset \Gamma$, where $R_{+}^{\times}$denotes the group of matrices in $R^{\times}$with positive determinant.

Lemma 3.3 For all $\gamma \in \iota\left(R_{+}^{\times}\right)$,

$$
f_{\gamma e}(\gamma z) d(\gamma z)=w^{|\gamma|_{p}} f_{e}(z) d z .
$$

The proof is explained in lemma 1.5 of [Da].

\section{Complex periods and Heegner points}

Recall that if $\kappa$ is a $\mathbb{Z}$-valued harmonic cocycle on $\mathcal{T}_{p}$, and $f$ is the associated rigid analytic function on $\mathcal{H}_{p}$, then by lemma 2.5 :

$$
\operatorname{ord}_{p}\left(\oint_{\tau_{1}}^{\tau_{2}} f(z) d z\right)=\sum_{e: r\left(\tau_{1}\right) \rightarrow r\left(\tau_{2}\right)} \kappa(e)
$$

for $\tau_{1}$ and $\tau_{2}$ in $\mathcal{H}_{p}^{\mathrm{nr}}$. In light of the definitions in the previous section, a natural notion of $\mathbb{C}$-valued line integral of an analytic function on $\mathcal{H}_{p} \times \mathcal{H}$ comes out of imagining an integration theory taking values in $\mathbb{C}_{p}^{\times} \otimes \mathbb{C}$ and applying to this hypothetical integral the map ord $: \mathbb{C}_{p}^{\times} \otimes \mathbb{C} \longrightarrow \mathbb{C}$. Thus, given $f \in S_{2}\left(\left(\mathcal{H}_{p} \times \mathcal{H}\right) / \Gamma\right)$, define the additive integral of this section by the rule:

$$
\int_{\tau_{1}}^{\tau_{2}} \int_{\tau_{3}}^{\tau_{4}} \omega_{f}=\sum_{e: r\left(\tau_{1}\right) \rightarrow r\left(\tau_{2}\right)} \int_{\tau_{3}}^{\tau_{4}} 2 \pi i f_{e}(z) d z
$$


for $\tau_{1}, \tau_{2} \in \mathcal{H}_{p}^{\mathrm{nr}}$ and $\tau_{3}, \tau_{4} \in \mathcal{H}^{*}$. It is immediately apparent that this double integral is additive in both sets of variables of integration, and satisfies the $\Gamma$-invariance property:

$$
\int_{\gamma \tau_{1}}^{\gamma \tau_{2}} \int_{\gamma \tau_{3}}^{\gamma \tau_{4}} \omega_{f}=\int_{\tau_{1}}^{\tau_{2}} \int_{\tau_{3}}^{\tau_{4}} \omega_{f} \quad \text { for all } \gamma \in \Gamma
$$

Having now a suitable notion of weight 2 cusp form on $\mathcal{H}_{p} \times \mathcal{H}$ and a theory of integration of such forms, one may begin to carry out the program described at the end of section 1 by defining a period attached to a quadratic subalgebra $K$ of $M_{2}(\mathbb{Q})$.

Let $\Gamma$ act trivially on $\mathbb{C}$. A direct computation shows that the function $\tilde{d}_{x, \tau}: \Gamma \times \Gamma \rightarrow \mathbb{C}$ defined by

$$
\tilde{d}_{x, \tau}\left(\gamma_{1}, \gamma_{2}\right)=\int_{\gamma_{1} x}^{\gamma_{1} \gamma_{2} x} \int_{\tau}^{\gamma_{1} \tau} \omega_{f}
$$

is a 2-cocycle, for all $x \in \mathcal{H}_{p}$ and $\tau \in \mathcal{H}$. Furthermore, the image of $\tilde{d}_{x, \tau}$ in $H^{2}(\Gamma, \mathbb{C})$ is independent of the base points $x \in \mathcal{H}_{p}$ and $\tau \in \mathcal{H}$ that were chosen to define it.

Assume for simplicity that the cusp form

$$
f_{0}:=f_{e^{o}} \in S_{2}^{p-\text { new }}\left(\Gamma_{0}(N)\right)
$$

is attached to an elliptic curve $E / \mathbb{Q}$ of conductor $N$, so that in particular $f_{0}$ has integer Fourier coefficients. Let $\Lambda \subset \mathbb{C}$ be the image of the relative homology $H_{1}\left(X_{0}(N)\right.$, cusps; $\left.\mathbb{Z}\right)$ under the integration pairing with $f_{0}$. The following theorem plays a crucial role in the definition of the period integral $J_{\Psi}$ defined in this section, as well as in a key construction of section 5 .

Theorem 4.1 (Manin-Drinfeld) The subgroup $\Lambda \subset \mathbb{C}$ is a lattice which is commensurable with the Néron lattice $\Lambda_{E}$ attached to $E$.

Skech of proof: The key to this proof is the fact that, for all primes $\ell$ not dividing $N$, the correspondences $\ell+1-T_{\ell}$ map the relative homology $H_{1}\left(X_{0}(N)\right.$, cusps; $\left.\mathbb{Z}\right)$ to $H_{1}\left(X_{0}(N), \mathbb{Z}\right)$. This implies that, for any such $\ell,\left(\ell+1-a_{\ell}\right) \Lambda$ is contained in $\Lambda_{E}$. The reader is refered to [Man1] or [Maz] where the proof is explained in more detail.

The lattice $\Lambda$ plays a key role in the integration theory considered in this section, thanks to the following lemma. 
Lemma 4.2 Let $f$ be the cusp form of weight 2 on $\mathcal{H}_{p} \times \mathcal{H}$ attached to $E$, and let $\Lambda$ be the lattice associated to $E$ as above.

1. The complex line integral $\int_{x}^{y} f_{e}(z) d z$ belongs to $\Lambda$, for all $x, y \in \mathbb{P}_{1}(\mathbb{Q})$ and all $e \in \mathcal{E}\left(\mathcal{T}_{p}\right)$.

2. For all $\tau_{1}, \tau_{2} \in \mathcal{H}_{p}^{\mathrm{nr}}$ and for all $x, y \in \mathbb{P}_{1}(\mathbb{Q})$,

$$
\int_{\tau_{1}}^{\tau_{2}} \int_{x}^{y} \omega_{f} \text { belongs to } \Lambda \text {. }
$$

Proof: To show the first part, note that if $e$ is any edge of $\mathcal{T}_{p}$, there exists an element $\gamma$ in $\Gamma$ such that

$$
\gamma^{-1} e^{o}=e \quad \text { or } \quad \gamma^{-1} e^{o}=\bar{e} .
$$

In any case

$$
\int_{x}^{y} f_{e}(z) d z= \pm \int_{\gamma x}^{\gamma y} f_{0}(z) d z
$$

and the result follows from the definition of $\Lambda$. The second part is an immediate consequence of the first, in light of the definition of the double integral attached to $f$.

Part 2 of lemma 4.2 is used in this section in the proof of the following proposition:

Proposition 4.3 The natural image of $\tilde{d}_{x, \tau}$ in $H^{2}(\Gamma, \mathbb{C} / \Lambda)$ is zero.

Proof: The proof is obtained by showing that the 2-cocycle $\tilde{d}_{x, \tau}$ viewed modulo $\Lambda$ is the image by the coboundary map of an explicit 1-cochain. Defining $\xi: \Gamma \rightarrow \mathbb{C}$ by

$$
\xi(\gamma)=\int_{x}^{\gamma x} \int_{\infty}^{\tau} \omega_{f}
$$

and using the fact that $\Gamma$ acts trivially on $\mathbb{C}$, one finds

$$
\begin{aligned}
d \xi\left(\gamma_{1}, \gamma_{2}\right) & =\int_{x}^{\gamma_{1} x} \int_{\infty}^{\tau} \omega_{f}+\int_{x}^{\gamma_{2} x} \int_{\infty}^{\tau} \omega_{f}-\int_{x}^{\gamma_{1} \gamma_{2} x} \int_{\infty}^{\tau} \omega_{f} \\
& =\int_{\gamma_{1} \gamma_{2} x}^{\gamma_{1} x} \int_{\infty}^{\tau} \omega_{f}+\int_{x}^{\gamma_{2} x} \int_{\infty}^{\tau} \omega_{f} \\
& =\int_{\gamma_{1} \gamma_{2} x}^{\gamma_{1} x} \int_{\infty}^{\tau} \omega_{f}+\int_{\gamma_{1} x}^{\gamma_{1} \gamma_{2} x} \int_{\gamma_{1} \infty}^{\gamma_{1} \tau} \omega_{f} \\
& =\int_{\gamma_{1} x}^{\gamma_{1} \gamma_{2} x} \int_{\tau}^{\gamma_{1} \tau} \omega_{f}+\int_{\gamma_{1} x}^{\gamma_{1} \gamma_{2} x} \int_{\gamma_{1} \infty}^{\infty} \omega_{f} .
\end{aligned}
$$


The second term of this last expression belongs to $\Lambda$ by part 2 of lemma 4.2 , and the result follows.

The 1-cochain $\xi$ is determined by $x$ and $\tau$ up to 1-cocycles. To make $\xi$ completely canonical one shows that the group of 1-cocyles has finite exponent, so that the ambiguity in the definition of $\xi$ can be removed by replacing $\xi$ by an appropriate integer multiple.

Lemma 4.4 The group of 1 -cocycles on $\Gamma$ with coefficients in $\mathbb{C} / \Lambda$ has finite exponent.

Proof: Since $\Gamma$ acts trivially on $\mathbb{C} / \Lambda$, the group of 1-cocycles on $\Gamma$ with coefficients in $\mathbb{C} / \Lambda$ is given by $\operatorname{Hom}(\Gamma, \mathbb{C} / \Lambda)=\operatorname{Hom}\left(\Gamma^{\mathrm{ab}}, \mathbb{C} / \Lambda\right)$. But the abelianisation $\Gamma^{\mathrm{ab}}$ of $\Gamma$ is finite by the corollary to theorem 3 in [Se2].

Let $\mathcal{O}$ be as in section 2 a $\mathbb{Z}[1 / p]$-order in an imaginary quadratic field $K$. Let $\mathcal{O}_{0}:=\mathcal{O} \cap \mathcal{O}_{K}$ be the maximal $\mathbb{Z}$-order in $\mathcal{O}$, and assume as in section 1 that the level $N$ satisfies the Heegner hypothesis, choosing as earlier a factorisation $N \mathcal{O}_{0}=\mathcal{N} \overline{\mathcal{N}}$ into a product of cyclic ideals of $K$. Set

$$
R:=\mathrm{M}_{0}(M) \otimes \mathbb{Z}[1 / p],
$$

where the $\mathbb{Z}[1 / p]$-algebra $R$ is viewed naturally as a subring of $M_{2}(\mathbb{Q})$. An oriented optimal embedding of $\mathcal{O}$ into $R$ is defined exactly as in section 1 , except that $N$ is replaced with $M, \mathcal{N}$ with $\mathcal{M}:=\mathcal{N O}$ and $M_{0}(N)$ with $R$, and the following additional orientation condition at $p$ is imposed. One asks that the normalized fixed point $\tau=\tau_{\Psi}$ for the action of $\Psi\left(K_{p}^{\times}\right)$on $\mathbb{P}_{1}\left(\mathbb{Q}_{p}\right)$ belongs to $\mathbb{Z}_{p}$, with respect to a fixed embedding of $K$ into $\mathbb{Q}_{p}$. Define $\operatorname{Emb}(\mathcal{O}, R)$ as earlier, with $\Gamma=R_{1}^{\times}$replacing $\Gamma_{0}(N)$. Let $\Psi \in \operatorname{Emb}(\mathcal{O}, R)$ be an oriented optimal embedding. Note that $\Psi\left(K_{p}^{\times}\right)$leaves invariant the geodesic on $\mathcal{T}_{p}$ joining the two fixed points of $\Psi\left(K_{p}^{\times}\right)$in $\mathbb{P}_{1}\left(\mathbb{Q}_{p}\right)$. Let $\Gamma_{\tau}$ denote the stabiliser of $\tau$ in $\Gamma$.

Lemma 4.5 The group $\Gamma_{\tau}$ has rank one.

Proof: Since $\Psi\left(K^{\times}\right)$is the stabiliser of $\tau$ in $\mathrm{GL}_{2}(\mathbb{Q})$ and $\Psi$ is an optimal embedding of $\mathcal{O}$ into $R$, it follows that $\Gamma_{\tau}=\Psi\left(\mathcal{O}_{1}^{\times}\right)$. The Dirichlet unit theorem shows that $\mathcal{O}_{1}^{\times}$is of rank one, with generator given by an appropriate power of the fundamental $p$-unit of the imaginary quadratic field $K$. 
Choose a generator $u$ of $\mathcal{O}_{1}^{\times}$and set $\gamma_{\tau}=\Psi(u)$. The calculations

$$
\begin{aligned}
\int_{x}^{\gamma_{\tau} x} \int_{\infty}^{\tau} \omega_{f}-\int_{y}^{\gamma_{\tau} y} \int_{\infty}^{\tau} \omega_{f} & =\int_{\gamma_{\tau} y}^{\gamma_{\tau} x} \int_{\infty}^{\tau} \omega_{f}-\int_{y}^{x} \int_{\infty}^{\tau} \omega_{f} \\
& =\int_{y}^{x} \int_{\gamma_{\tau}^{-1} \infty}^{\tau} \omega_{f}-\int_{y}^{x} \int_{\infty}^{\tau} \omega_{f}=0 \quad(\bmod \Lambda),
\end{aligned}
$$

and

$$
\int_{x}^{\gamma \gamma_{\tau} \gamma^{-1} x} \int_{\infty}^{\gamma \tau} \omega_{f}=\int_{\gamma^{-1} x}^{\gamma_{\tau} \gamma^{-1} x} \int_{\gamma^{-1} \infty}^{\tau} \omega_{f}=\int_{x}^{\gamma_{\tau} x} \int_{\infty}^{\tau} \omega_{f} \quad(\bmod \Lambda),
$$

show that $\xi\left(\gamma_{\tau}\right)$ depends only on the $\Gamma$-conjugacy class of $\Psi$, and not on the choice of $x$, and later of $\xi$, that were made to define it. Define

$$
J_{\Psi}:=\xi\left(\gamma_{\tau}\right)=\sum_{e: v \rightarrow \gamma_{\tau} v} \int_{\infty}^{\tau} 2 \pi i f_{e}(t) d t
$$

where $v$ is any choice of vertex. Note that the rightmost term in (37) is independent of the choice of $v$.

Write $p \mathcal{O}_{0}=\mathfrak{p} \overline{\mathfrak{p}}$ and let $\varpi$ be a uniformizer for $\mathfrak{p} \otimes \mathbb{Z}_{p}=\varpi\left(\mathcal{O}_{0} \otimes \mathbb{Z}_{p}\right)$.

Lemma 4.6 Any class $\Psi \in \operatorname{Emb}(\mathcal{O}, R)$ has a representative $\Psi_{0}$ whose restriction to $\mathcal{O}_{0}$ is an oriented optimal embedding (in the sense of section 1) of $\mathcal{O}_{0}$ into $M_{0}(N)$ characterized by the property that $v^{o}$ and $\Psi(\varpi) v^{o}$ are adjacent vertices connected by $e^{o}$.

Proof. Let $e$ be any edge of $\mathcal{T}_{p}$ that lies on the path joining the two fixed points of $\Psi\left(K_{\mathfrak{p}}^{\times}\right)$acting on $\mathbb{P}_{1}\left(\mathbb{Q}_{p}\right)$. By interchanging the fixed points if necessary, there exists an element $\gamma \in \Gamma$ such that $\gamma e=e^{o}$. The embedding $\Psi_{0}:=\gamma \Psi \gamma^{-1}$ yields the desired optimal embedding of $\mathcal{O}_{0}$ into $M_{0}(N)$. The lattice $\Psi(\varpi) \cdot \mathbb{Z}_{p}^{2}$ is homothetic to $\mathbb{Z}_{p}\left(\begin{array}{c}\tau_{\Psi_{0}} \\ 1\end{array}\right)+\mathbb{Z}_{p}\left(\begin{array}{c}p \\ 0\end{array}\right)$ which is in the class of the vertex adjacent $v^{o}$ on the end to $\tau_{\Psi_{0}}$, and so the result follows.

For $\Psi_{0} \in \operatorname{Emb}\left(\mathcal{O}_{0}, \mathrm{M}_{0}(N)\right)$, let $J_{0, \Psi_{0}}$ denote the period attached to it in section 1 (where it was denoted $J_{\Psi_{0}}$ ). Recall the sign $w$ introduced before the statement of lemma 3.3, and let $t$ be twice the order of $\mathfrak{p}^{2}$ in $\operatorname{Pic}\left(\mathcal{O}_{0}\right)$. 
Proposition 4.7 Suppose $\Psi \in \operatorname{Emb}(\mathcal{O}, R)$ and let $\Psi_{0} \in \operatorname{Emb}\left(\mathcal{O}_{0}, \mathrm{M}_{0}(N)\right)$ be as in lemma 4.6. Then

$$
J_{\Psi}=\sum_{j=0}^{t-1} w^{j} J_{0, \Psi_{0}^{\mathfrak{p} j}}(\bmod \Lambda) .
$$

Proof: Let $v_{0}, v_{1}, \ldots, v_{t}$ denote the consecutive vertices on the path joining $v_{0}$ to $v_{t}:=\gamma_{\Psi} v_{0}$, and let $e_{j}$ (for $0 \leq j \leq t-1$ ) denote the edge joining $v_{j}$ to $v_{j+1}$. Let $R_{v_{0}, v_{t}}$ denote the subring of $M_{2}\left(\mathbb{Q}_{p}\right)$ consisting of the matrices which fix both $v_{0}$ and $v_{t}$. It is a local Eichler order of level $t$ in $M_{2}\left(\mathbb{Z}_{p}\right)$, and it fixes pointwise all the vertices $v_{0}, \ldots, v_{t}$ as well as the edges $e_{0}, \ldots, e_{t-1}$. Note that

$$
\Psi(\varpi)\left(v_{j}\right)=v_{j+1}, \quad \text { for } j=0, \ldots, t .
$$

By strong approximation, there exists an element $\gamma_{*} \in R_{+}^{\times}$whose image in $\mathbf{P G L}_{2}\left(\mathbb{Q}_{p}\right) / R_{v_{0}, v_{t}}^{\times}$is equal to that of $\Psi(\varpi)$. In particular,

$$
\gamma_{*}\left(e_{j}\right)=e_{j+1}, \quad \text { for } j=0, \ldots, t-1 .
$$

By definition,

$$
J_{\Psi}=\sum_{j=0}^{t-1} \int_{\infty}^{\tau_{\Psi_{0}}} f_{e_{j}}(z) d z \quad(\bmod \Lambda)
$$

But

$$
\int_{\infty}^{\tau_{\Psi_{0}}} f_{e_{j}}(z) d z=\int_{\infty}^{\tau_{\Psi_{0}}} f_{\gamma_{*}^{j} e_{0}}(z) d z=w^{j} \int_{\infty}^{\gamma_{*}^{-j} \tau_{\Psi_{0}}} f_{0}(z) d z \quad(\bmod \Lambda),
$$

where the last equality follows from lemma 3.3. On the other hand, by the description of the action of $\operatorname{Pic}\left(\mathcal{O}_{0}\right)$ on $\operatorname{Emb}\left(\mathcal{O}_{0}, R_{0}\right)$ given in lemma 1.3, we have

$$
\int_{\infty}^{\gamma_{*}^{-j} \tau_{\Psi_{0}}} f_{0}(z) d z=J_{0, \Psi_{o}^{\mathrm{p} j}}
$$

The proposition follows by combining equations (38), (39) and (40).

Let $H_{0}$ denote the ring class field of $K$ attached to the $\mathbb{Z}$-order $\mathcal{O}_{0}$, and let $H:=H_{0}^{\text {Frob }_{\mathfrak{p}}^{2}}$ be the " $p$-narrow" class field. The reciprocity map of class field theory gives a canonical identification

$$
\text { rec }: \operatorname{Pic}^{p+}(\mathcal{O}) \longrightarrow \operatorname{Gal}(H / K)
$$


where $\operatorname{Pic}^{p+}(\mathcal{O})$ is defined just as the usual class group $\operatorname{Pic}(\mathcal{O})$, except that equivalence is only up to scalars whose norm has even valuation at $p$. Letting $\eta$ be the Weierstrass uniformisation of $E$ as before, one obtains the following analogue of theorem 1.4:

Theorem 4.8 For all $\Psi$ in $\operatorname{Emb}(\mathcal{O}, R)$, the point $\eta\left(J_{\Psi}\right)$ belongs to $E(H)$. More precisely, for all $\mathfrak{b} \in \mathrm{Pic}^{p+}(\mathcal{O})$,

$$
\eta\left(J_{\Psi^{\mathfrak{b}}}\right)=\operatorname{rec}(\mathfrak{b})^{-1} \cdot \eta\left(J_{\Psi}\right) .
$$

It is worth noting that proposition 4.7 implies that

$$
\eta\left(J_{\Psi}\right)^{\operatorname{Frob}_{p}}=w \eta\left(J_{\Psi}\right),
$$

so that the global point $\eta\left(J_{\Psi}\right)$ belongs to the $w$-eigenspace for the involution Frob $_{p}$ acting on $E(H)$.

\section{$5 \quad p$-adic periods and Stark-Heegner points}

We now modify the discussion of the previous section by interchanging the roles of the places $p$ and $\infty$.

The first stage in carrying out this program is to replace the $\mathbb{C}$-valued double integral of equation (34) by a $\mathbb{C}_{p}$-valued integral

$$
\int_{\tau_{1}}^{\tau_{2}} \int_{\tau_{3}}^{\tau_{4}} \omega_{f} \stackrel{?}{\in} \mathbb{C}_{p} \quad\left(\tau_{1}, \tau_{2} \in \mathcal{H}_{p}, \quad \tau_{3}, \tau_{4} \in \mathcal{H}^{*}\right) .
$$

A natural way of doing so is to start by using the complex endpoints of integration $\tau_{3}, \tau_{4}$ to convert the form-valued harmonic cocycle $e \mapsto f_{e}$ of section 4 into a $\mathbb{C}$-valued harmonic cocycle $\tilde{\kappa}_{f}\left\{\tau_{3} \rightarrow \tau_{4}\right\}$ on $\mathcal{T}_{p}$ by the rule:

$$
\tilde{\kappa}_{f}\left\{\tau_{3} \rightarrow \tau_{4}\right\}(e):=\int_{\tau_{3}}^{\tau_{4}} f_{e}(z) d z .
$$

The resulting distribution, denoted $\tilde{\mu}_{f}\left\{\tau_{3} \rightarrow \tau_{4}\right\}$, can be meaningfully integrated against locally constant $\mathbb{C}$-valued functions on $\mathbb{P}_{1}\left(\mathbb{Q}_{p}\right)$. Taking one's cue from equation (13) of section 2 defining Coleman's $p$-adic line integral, it would be tempting to define

$$
\int_{\tau_{1}}^{\tau_{2}} \int_{\tau_{3}}^{\tau_{4}} \omega_{f}: \stackrel{?}{=} \int_{\mathbb{P}_{1}\left(\mathbb{Q}_{p}\right)} \log \left(\frac{t-\tau_{2}}{t-\tau_{1}}\right) d \tilde{\mu}_{f}\left\{\tau_{3} \rightarrow \tau_{4}\right\}(t),
$$


were it not for the fact that the integrand is $\mathbb{C}_{p}$ and not $\mathbb{C}$-valued, and is merely locally analytic, not locally constant. To make sense of (43), it is necessary to modify the definition of the complex distribution $\tilde{\mu}_{f}\left\{\tau_{3} \rightarrow \tau_{4}\right\}$ so that its values become $p$-adic, and bounded. This can be achieved, at the cost of restricting the complex endpoints $\tau_{3}, \tau_{4}$ to lie in the boundary $\mathbb{P}_{1}(\mathbb{Q})$ of the extended Poincaré upper half plane $\mathcal{H}^{*}$. Once this is done, the distribution $\tilde{\mu}_{f}\left\{\tau_{3} \rightarrow \tau_{4}\right\}$ acquires the desired integrality properties, since it arises from a cocycle that takes values in the $\mathbb{Z}$-lattice $\Lambda$ that occurs in the statement of theorem 4.1 of section 4. (Cf. part 1 of lemma 4.2.)

Motivated by this lemma, assume henceforth that $\tau_{3}, \tau_{4}$ belong to $\mathbb{P}_{1}(\mathbb{Q})$, so that $\tilde{\mu}_{f}\left\{\tau_{3} \rightarrow \tau_{4}\right\}$ can be viewed as a $\Lambda$-valued $p$-adic measure.

For the sake of notational simplicity, let $\beta: \Lambda \longrightarrow \mathbb{Z}$ be the surjective group homomorphism which is zero on $(i \mathbb{R} \cap \Lambda)$ and maps positive real numbers in $(\Lambda \cap \mathbb{R})$ to positive integers. (It may be noted that any other choice of $\beta$ will do equally well for our purposes.) Using $\beta$, define a $\mathbb{Z}_{p}$-valued measure on $\mathbb{P}_{1}\left(\mathbb{Q}_{p}\right)$ by the rule

$$
\mu_{f}\left\{\tau_{3} \rightarrow \tau_{4}\right\}(U):=\beta\left(\tilde{\mu}_{f}\left\{\tau_{3} \rightarrow \tau_{4}\right\}(U)\right) .
$$

Equation (43), with $\tilde{\mu}_{f}$ replaced by $\mu_{f}$, now makes perfect sense. Because

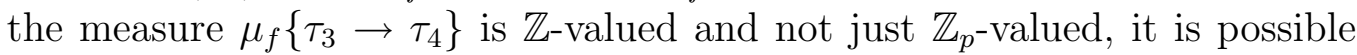
to apply the same multiplicative refinement as in the definition (13) of the multiplicative Coleman integral of section 2 to define

$$
\oint_{\tau_{1}}^{\tau_{2}} \int_{\tau_{3}}^{\tau_{4}} \omega_{f}:=\oint_{\mathbb{P}_{1}\left(\mathbb{Q}_{p}\right)}\left(\frac{t-\tau_{2}}{t-\tau_{1}}\right) d \mu_{f}\left\{\tau_{3} \rightarrow \tau_{4}\right\}(t) .
$$

Mimicking the definitions in section 4 , let $\tilde{d}_{\tau, x} \in Z^{2}\left(\Gamma, \mathbb{C}_{p}^{\times}\right)$be the two-cocycle obtained as in equation (36) by choosing base points $\tau \in \mathcal{H}_{p}$ and $x \in \mathbb{P}_{1}(\mathbb{Q})$ and setting

$$
\tilde{d}_{\tau, x}\left(\gamma_{1}, \gamma_{2}\right):=\oiint_{\tau}^{\gamma_{1} \tau} \int_{\gamma_{1} x}^{\gamma_{1} \gamma_{2} x} \omega_{f}
$$

Let $d \in H^{2}\left(\Gamma, \mathbb{C}_{p}^{\times}\right)$be the cohomology class of $\tilde{d}_{\tau, x}$. A direct calculation shows that this class does not depend on the choices of base points $\tau$ and $x$ that were made to define $\tilde{d}_{\tau, x}$.

It will be convenient in some of the calculations to relate the class $d$ to an element in the first cohomology of $\Gamma$ with values in a module of so-called $M$ symbols. If $A$ is any abelian group, a function $m\{\}:, \mathbb{P}_{1}(\mathbb{Q}) \times \mathbb{P}_{1}(\mathbb{Q}) \longrightarrow A$ 
denoted $(x, y) \mapsto m\{x \rightarrow y\}$ is called an (A-valued) $M$-symbol if it satisfies

$$
m\{x \rightarrow y\}+m\{y \rightarrow z\}=m\{x \rightarrow z\},
$$

for all $x, y, z \in \mathbb{P}_{1}(\mathbb{Q})$. Let $\mathcal{M}(A)$ (resp. $\mathcal{F}(A)$ ) denote the left $\Gamma$-module of $A$-valued $M$-symbols (resp. functions) on $\mathbb{P}_{1}(\mathbb{Q})$. In the special case where $A=\mathbb{C}_{p}$, it is suppressed from the notations, so that $\mathcal{M}$ and $\mathcal{F}$ denote the modules of $\mathbb{C}_{p}$ valued $M$-symbols and functions respectively.

The map $\Delta: \mathcal{F} \longrightarrow \mathcal{M}$ defined by

$$
(\Delta f)\{x \rightarrow y\}:=f(y)-f(x)
$$

is surjective and has as kernel the space of constant functions. Taking the cohomology of the short exact sequence of $\mathbb{C}_{p}[\Gamma]$-modules

$$
0 \longrightarrow \mathbb{C}_{p} \longrightarrow \mathcal{F} \stackrel{\Delta}{\longrightarrow} \mathcal{M} \longrightarrow 0
$$

yields a long exact sequence in cohomology:

$$
H^{1}(\Gamma, \mathcal{F}) \longrightarrow H^{1}(\Gamma, \mathcal{M}) \stackrel{\delta}{\longrightarrow} H^{2}\left(\Gamma, \mathbb{C}_{p}\right) \longrightarrow H^{2}(\Gamma, \mathcal{F})
$$

and likewise with $\mathbb{C}_{p}$ replaced by any abelian group $A$.

If one fixes $\tau \in \mathcal{H}_{p}$ and defines

$$
\tilde{c}_{\tau}(\gamma)\{x \rightarrow y\}:=\aleph_{\tau}^{\gamma \tau} \int_{x}^{y} \omega_{f}
$$

then $\tilde{c}_{\tau}$ is an $\mathcal{M}\left(\mathbb{C}_{p}^{\times}\right)$-valued one-cocycle. A direct computation shows that its class $c \in H^{1}\left(\Gamma, \mathcal{M}\left(\mathbb{C}_{p}^{\times}\right)\right)$is independent of the choice of $\tau$ and satisfies

$$
\delta(c)=d .
$$

As is explained in [Da], sec. 3.1, the cohomology groups $H^{1}(\Gamma, \mathcal{M})$ and $H^{2}\left(\Gamma, \mathbb{C}_{p}\right)$ are finite-dimensional $\mathbb{C}_{p}$-vector spaces equipped with an action of the algebra $\mathbb{T}$ of Hecke operators as well as an action of an involution $W_{\infty}$ induced by conjugation by the matrix $\left(\begin{array}{cc}1 & 0 \\ 0 & -1\end{array}\right)$. Let $H^{1}(\Gamma, \mathcal{M})^{f,+}$ denote the $f$ isotypic part of $H^{1}(\Gamma, \mathcal{M})$ fixed by $W_{\infty}$, and similarly for $H^{2}\left(\Gamma, \mathbb{C}_{p}\right)$.

Lemma 5.1 The groups $H^{1}(\Gamma, \mathcal{M})^{f,+}$ and $H^{2}\left(\Gamma, \mathbb{C}_{p}\right)^{f,+}$ are one-dimensional $\mathbb{C}_{p}$-vector spaces. 
Proof: All the maps in the exact sequence (48) are equivariant under the natural actions of the Hecke operators and under the action of the involution $W_{\infty}$. Furthemore, as explained in [Da], sec. 3.1, the modules $H^{j}(\Gamma, \mathcal{F})^{f,+}$ are trivial. Hence $\delta$ induces an isomorphism

$$
\delta: H^{1}(\Gamma, \mathcal{M})^{f,+} \longrightarrow H^{2}\left(\Gamma, \mathbb{C}_{p}\right)^{f,+} .
$$

The result now follows from [Da], corollary 3.3.

Note that the assumption that $p$ divides $N$ exactly implies that $E$ has (either split or non-split) multiplicative reduction at $p$, so that it is equipped with the Tate $p$-adic uniformisation

$$
\eta_{p}: \mathbb{C}_{p}^{\times} / q^{Z} \longrightarrow E\left(\mathbb{C}_{p}\right)
$$

where $q \in p \mathbb{Z}_{p}$ is Tate's $p$-adic period attached to $E_{/ \mathbb{Q}_{p}}$. Let $w= \pm 1$ be the sign that was introduced earlier: thus $w=1$ if $E$ has split multiplicative reduction at $p$, and $w=-1$ if $E$ has non-split multiplicative reduction.

The following proposition, referred to from now on as the $p$-adic period theorem, is a natural analoge of proposition 4.3 (combined with theorem 4.1) of section 4 .

Theorem 5.2 There exists a lattice $\Lambda_{p} \subset \mathbb{C}_{p}^{\times}$which is commensurable with $q^{\mathbb{Z}}$, and such that the natural images of $d$ in $H^{2}\left(\Gamma, \mathbb{C}_{p}^{\times} / \Lambda_{p}\right)$ and of $c$ in $H^{1}\left(\Gamma, \mathcal{M}\left(\mathbb{C}_{p}^{\times} / \Lambda_{p}\right)\right)$ are trivial.

Sketch of proof: Let $\operatorname{ord}_{p}: \mathbb{C}_{p}^{\times} \longrightarrow \mathbb{Q} \subset \mathbb{C}_{p}$ be the usual normalised $p$-adic valuation and let $\log _{p}: \mathbb{C}_{p}^{\times} \longrightarrow \mathbb{C}_{p}$ be a branch of the $p$-adic logarithm, chosen, to fix ideas, in such a way that $\log _{p}(p)=0$. The one-cocycles $\operatorname{ord}_{p}(c)$ and $\log _{p}(c)$ each belong to $H^{1}(\Gamma, \mathcal{M})^{f,+}$, and $\operatorname{ord}_{p}(c) \neq 0$ (cf. [Da], lemma 3.4). By lemma 5.1, there exists a constant $\mathcal{L}$ in $\mathbb{C}_{p}$ such that

$$
\log _{p}\left(\tilde{c}_{\tau}\right)=\mathcal{L} \cdot \operatorname{ord}_{p}\left(\tilde{c}_{\tau}\right) \quad\left(\bmod B^{1}(\Gamma, \mathcal{M})\right)
$$

To evaluate the constant $\mathcal{L}$, let $n$ be any positive integer which is relatively prime to $N$ and let $j$ be the least integer such that $p^{2 j} \equiv 1(\bmod n)$. If $\nu$ is in $(\mathbb{Z} / n \mathbb{Z})^{\times}$, let $\gamma_{\nu}:=\left(\begin{array}{cc}p^{j} & \nu \frac{p^{j}-p^{-j}}{n} \\ 0 & p^{-j}\end{array}\right)$ be the generator of the stabiliser in $\Gamma$ of

$$
(\infty, \nu / c) \in \mathbb{P}_{1}(\mathbb{Q}) \times \mathbb{P}_{1}(\mathbb{Q})
$$


Note that for all 1-coboundaries $b \in B^{1}(\Gamma, \mathcal{M})$,

$$
b\left(\gamma_{\nu}\right)\{\infty \rightarrow \nu / n\}=0
$$

Hence for all $\nu \in(\mathbb{Z} / n \mathbb{Z})^{\times}$, equation (50) implies

$$
\log \left(\tilde{c}_{\tau}\right)\left(\gamma_{\nu}\right)\{\infty \rightarrow \nu / n\}=\mathcal{L} \cdot \operatorname{ord}_{p}\left(\tilde{c}_{\tau}\right)\left(\gamma_{\nu}\right)\{\infty \rightarrow \nu / n\} .
$$

Let $\chi:(\mathbb{Z} / n \mathbb{Z})^{\times} \longrightarrow \mathbb{C}_{p}^{\times}$be a Dirichlet character of conductor $n$, viewed as taking values in $\mathbb{C}_{p}$, and suppose that $\chi(p)=w$, and that $\chi(-1)=1$. In [Da], prop. 2.16, it is shown that

$$
\sum_{\nu \in(\mathbb{Z} / n \mathbb{Z})^{\times}} \chi(\nu) \operatorname{ord}_{p}\left(\tilde{c}_{\tau}\right)\left(\gamma_{\nu}\right)\{\infty \rightarrow \nu / n\}=\frac{2 j n}{\tau(\chi)} \frac{L(E / \mathbb{Q}, \chi, 1)}{\Omega^{+}},
$$

where $\tau(\chi)$ is the Gauss sum attached to $\chi$ and $\Omega^{+}$is an appropriate rational multiple of the real period of $E$. An independent calculation, which follows by combining proposition 2.18 of [Da] with the definition of the MazurSwinnerton-Dyer $p$-adic $L$-function $L_{p}(E / \mathbb{Q}, s)$ attached to $E / \mathbb{Q}$ shows that

$$
\sum_{\nu \in(\mathbb{Z} / n \mathbb{Z})^{\times}} \chi(\nu) \log _{p}\left(\tilde{c}_{\tau}\right)\left(\gamma_{\nu}\right)\{\infty \rightarrow \nu / n\}=L_{p}^{\prime}(E / \mathbb{Q}, \chi, 1) .
$$

The two equations (52) and (53) combined with (51) imply that the discrepancy between the (algebraic part of the) classical $L$-value $L(E / \mathbb{Q}, \chi, 1)$ and the first derivative $L_{p}^{\prime}(E / \mathbb{Q}, \chi, 1)$, for even Dirichlet characters $\chi$ for which $\chi(p)=w$, is given by a constant $\mathcal{L}$ which is independent of $\chi$, a conjecture that was made by Mazur, Tate and Teitelbaum [MTT]. The article [MTT] went further than this, conjecturing on the basis of numerical evidence that

$$
\mathcal{L}=\frac{\log _{p}(q)}{\operatorname{ord}_{p}(q)}
$$

where $q$ is the Tate $p$-adic period attached to $E / \mathbb{Q}_{p}$. This conjecture was later proved by Greenberg and Stevens [GS]. This crucial result - which is the principal ingredient in the proof of theorem 5.2 - shows that

$$
\log (c)=\frac{\log _{p}(q)}{\operatorname{ord}_{p}(q)} \operatorname{ord}_{p}(c), \quad \log (d)=\frac{\log _{p}(q)}{\operatorname{ord}_{p}(q)} \operatorname{ord}_{p}(d),
$$


where the second equality follows from equation (49) relating the classes $c$ and $d$. Now consider the two-cocycle

$$
\tilde{e}_{\tau, x}:=\tilde{d}_{\tau, x}^{\operatorname{ord}_{p}(q)} \div q^{\operatorname{ord}_{p}\left(\tilde{d}_{\tau, x}\right)} \in Z^{2}\left(\Gamma, \mathbb{C}_{p}^{\times}\right) .
$$

and let $e$ be its natural image in $H^{2}\left(\Gamma, \mathbb{C}_{p}^{\times}\right)$. The second part of equation (55) implies that

$$
\operatorname{ord}_{p}(e)=\log _{p}(e)=0,
$$

so that $e$ is torsion. Hence there exists an integer constant $a$ such that

$$
\tilde{d}_{\tau, x}^{a \cdot \operatorname{ord}_{p}(q)}=q^{a \cdot \operatorname{ord}_{p}\left(\tilde{d}_{\tau, x}\right)} \quad\left(\bmod B^{2}\left(\Gamma, \mathbb{C}_{p}^{\times}\right)\right) .
$$

Theorem 5.2 follows.

Assume, at the cost of eventually raising $\tilde{c}_{\tau}$ and $\tilde{d}_{\tau, x}$ to a common integer power, that $\Lambda_{p}=q^{\mathbb{Z}}$. Thanks to theorem 5.2, one may as in section 4 define an $M$-symbol $m_{\tau} \in C^{0}\left(\Gamma, \mathcal{M}\left(\mathbb{C}_{p}^{\times} / q^{\mathbb{Z}}\right)\right)$ and a one-cochain $\xi_{\tau, x} \in C^{1}\left(\Gamma, \mathbb{C}_{p}^{\times} / q^{\mathbb{Z}}\right)$ by the rules

$$
\tilde{c}_{\tau}=d m_{\tau}, \quad \tilde{d}_{\tau, x}=d \xi_{\tau, x} .
$$

It is useful to adopt the notation

$$
\int^{\tau} \int_{x}^{y} \omega_{f}:=m_{\tau}\{x \rightarrow y\} \in \mathbb{C}_{p}^{\times} / q^{\mathbb{Z}}
$$

which can be justified by noting that this "semi-indefinite" integral satisfies the identities

$$
\begin{aligned}
& \mathcal{f}^{\tau} \int_{x}^{y} \omega_{f} \times \mathcal{f}^{\tau} \int_{y}^{z} \omega_{f}=\mathcal{f}^{\tau} \int_{x}^{z} \omega_{f} \text { for all } x, y, z \in \mathbb{P}_{1}(\mathbb{Q}), \\
& \varkappa^{\gamma \tau} \int_{\gamma x}^{\gamma y} \omega_{f}=\oint^{\tau} \int_{x}^{y} \omega_{f}, \quad \text { for all } \gamma \in \Gamma
\end{aligned}
$$

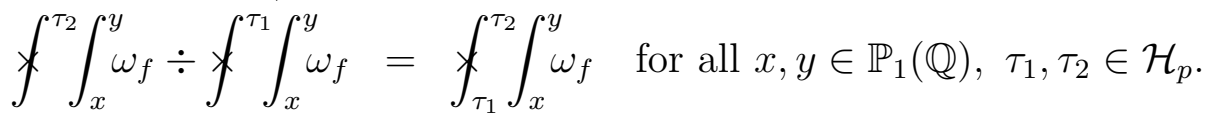

Let $\mathcal{O}$ be a $\mathbb{Z}[1 / p]$-order in the real quadratic field $K$. The notion of optimal embedding $\Psi: K \longrightarrow M_{2}(\mathbb{Q})$ is defined very much as in sections 2 and 4. I.e., we say that $\Psi$ is optimal if $\Psi(K) \cap R=\Psi(\mathcal{O})$.

One likewise defines a notion of oriented optimal embedding by introducing orientations on $\mathcal{O}$ and on $R$ (defined as ring homomorphisms to $\mathbb{Z} / M \mathbb{Z}$ ) 
and requiring that the embedding be compatible with these orientations. One requires in addition that the (unique) vertex on $\mathcal{T}_{p}$ fixed by $\Psi\left(K^{\times}\right)$is $\mathbf{S L}_{2}\left(\mathbb{Q}_{p}\right)$-equivalent to $v_{0}$, i.e., its distance from this vertex is even. (This last requirement can be viewed as a condition of "orientation at $p$ ".)

We can then define, just as in section 4 :

$$
J_{\Psi}:=\xi_{\tau, x}\left(\gamma_{\tau}\right)=\oint^{\tau} \int_{x}^{\gamma_{\tau} x} \omega_{f} \in \mathbb{C}_{p}^{\times} / q^{\mathbb{Z}} .
$$

Lemma 5.3 The period $J_{\Psi}$ depends only on the $\Gamma$-conjugacy class of $\tau$ (and therefore, of $\Psi)$, not on the choices of $x$ and then $\xi_{\tau, x}$ that were made to define it.

Proof. This follows from manipulations identical to those that were used to prove the corresponding statement in section 4 .

Let $H$ denote the narrow ring class field of $K$ attached to the order $\mathcal{O}$, (or, equivalently, to the $\mathbb{Z}$-order $\mathcal{O}_{0} \subset \mathcal{O}$, since the prime $p$ is inert in $K / \mathbb{Q}$ and hence splits completely in $H / K)$. The reciprocity map of class field theory gives a canonical identification

$$
\text { rec }: \operatorname{Pic}^{+}(\mathcal{O}) \longrightarrow \operatorname{Gal}(H / K) \text {. }
$$

Here $\operatorname{Pic}^{+}(\mathcal{O})$ is defined just as the usual class group $\operatorname{Pic}(\mathcal{O})$, except that two fractional $\mathcal{O}$-ideals are said to be equivalent if they differ by multiplication by an element of positive norm.

Let $\eta_{p}$ be the Tate uniformisation of $E$ of equation (20). The counterpart of theorem 4.8 in this setting is given by:

Conjecture 5.4 For all $\Psi$ in $\operatorname{Emb}(\mathcal{O}, R)$, the point $\eta_{p}\left(J_{\Psi}\right)$ belongs to $E(H)$. More precisely, for all $\mathfrak{b} \in \operatorname{Pic}^{+}(\mathcal{O})$,

$$
\eta\left(J_{\Psi^{\mathfrak{b}}}\right)=\operatorname{rec}(\mathfrak{b})^{-1} \cdot \eta\left(J_{\Psi}\right) .
$$

Note that, thanks to the change of notations and definitions entailed by our passage from a $\mathbb{C}$ to a $\mathbb{C}_{p}^{\times}$-valued integral and our change of quadratic field $K$, the statement of conjecture 5.4 is identical to that of theorem 4.8. Yet the former appears to lie deeper than the latter, and no proof is known for it. What is lacking is an analogue of the theory outlined in section 1, which was instrumental in proving theorem 4.8. 


\section{Heegner points and integration on $\mathcal{H}_{p} \times \mathcal{H}_{q}$}

This section considers an analogue of the conjecture formulated in section 5 , where one replaces the archimedean upper half plane $\mathcal{H}$ by a non-archimedean upper half plane $\mathcal{H}_{q}$. Here one is led to revisit the setting of section 2, since the group acting on the product $\mathcal{H}_{p} \times \mathcal{H}_{q}$ is defined in terms of a definite quaternion algebra $B$ which is split at $p$ and $q$. The period integral arises from a quadratic algebra $K$ which embeds in $B$ (so that $K$ is an imaginary quadratic field), such that $p$ is inert and $q$ is split in $K$. In this case the analogue of the above mentioned conjecture can be reduced to theorem 2.9 and therefore be proven.

Forms on $\mathcal{T}_{p} \times \mathcal{H}_{q}$

Assume that the conductor $N$ of $E$ can be written in the form

$$
N=p q N^{+} N^{-}
$$

where $p$ and $q$ are primes, the factors are pairwise relatively prime, and $N^{-}$is squarefree and divisible by an odd number of prime factors. It follows that $E$ has multiplicative reduction at $p$ and $q$. Moreover, the above factorisation is of the type (8) considered in section 2 , in which the integer currently denoted $N^{+} q$ was written $N^{+}$.

Let $B$ be the (definite) quaternion algebra over $\mathbb{Q}$ of discriminant $N^{-}$, let $R$ be an Eichler $\mathbb{Z}[1 / p, 1 / q]$-order in $B$ of level $N^{+}$, and let $R_{1}^{\times}$be the group of norm 1 elements in $R^{\times}$. Fix a embedding $\iota$ of $B$ into $M_{2}\left(\mathbb{Q}_{p}\right) \times M_{2}\left(\mathbb{Q}_{q}\right)$, and define a group

$$
\Gamma:=\iota\left(R_{1}^{\times}\right) \subset \mathbf{S L}_{2}\left(\mathbb{Q}_{p}\right) \times \mathbf{S L}_{2}\left(\mathbb{Q}_{q}\right) .
$$

Write $\gamma=\left(\gamma_{p}, \gamma_{q}\right)$ for the elements of $\Gamma$. The group $\Gamma$ acts discretely and properly discontinuously on the product $\mathcal{H}_{p} \times \mathcal{H}_{q}$ of the $p$-adic and $q$-adic upper half planes, by the rule $\gamma\left(\tau_{p}, \tau_{q}\right)=\left(\gamma_{p} \tau_{p}, \gamma_{q} \tau_{q}\right)$. It follows that $\Gamma$ acts naturally also on the product $\mathcal{E}\left(\mathcal{T}_{p}\right) \times \mathcal{H}_{q}$.

Definition 6.1 A cusp form of weight 2 on $\left(\mathcal{T}_{p} \times \mathcal{H}_{q}\right) / \Gamma$ is a function

$$
f: \mathcal{E}\left(\mathcal{T}_{p}\right) \times \mathcal{H}_{q} \longrightarrow \mathbb{C}_{q}
$$

satisfying 
1. $f\left(\gamma_{p} e, \gamma_{q} z\right)=(c z+d)^{2} f(e, z)$ for all $\gamma \in \Gamma$ with $\gamma_{q}=\left(\begin{array}{cc}a & b \\ c & d\end{array}\right)$.

2. For each $z \in \mathcal{H}_{q}$, the function $f_{z}(e):=f(e, z)$ is a $\mathbb{C}_{q}$-valued harmonic cocycle on $\mathcal{E}\left(\mathcal{T}_{p}\right)$.

3. For each $e \in \mathcal{E}\left(\mathcal{T}_{p}\right)$, the function $f_{e}(z):=f(e, z)$ is a q-adic rigid analytic modular form (see section 2) on the stabilizer $\Gamma_{e}$ of e in $\Gamma$.

Denote by $S_{2}\left(\left(\mathcal{T}_{p} \times \mathcal{H}_{q}\right) / \Gamma\right)$ the $\mathbb{C}_{q}$-vector space of weight 2 cusp forms on $\left(\mathcal{T}_{p} \times \mathcal{H}_{q}\right) / \Gamma$. Define a base edge $e^{o}$ in $\mathcal{E}\left(\mathcal{T}_{p}\right)$ by the property that $\Gamma_{e^{o}}$ be equal via $\iota$ to the group $\Gamma_{q}$ of norm 1 elements in a fixed Eichler $\mathbb{Z}[1 / q]$-order in $R$ of level $p N^{+}$. Denote by $S_{2}^{p-\text { new }}\left(\mathcal{H}_{q} / \Gamma_{q}\right)$ the subspace of weight 2 rigid analytic $q$-adic modular forms on $\Gamma_{q}$ which are new at $p$.

Lemma 6.2 The assignment sending $f$ to $f_{e^{\circ}}$ induces an isomorphism from $S_{2}\left(\left(\mathcal{T}_{p} \times \mathcal{H}_{q}\right) / \Gamma\right)$ to $S_{2}^{p-\text { new }}\left(\mathcal{H}_{q} / \Gamma_{q}\right)$.

Proof: The ideas in this proof are identical to those in the proof of proposition 3.2 of section 3 .

Note that the spaces appearing in lemma 6.2 are equipped with the natural action of a Hecke algebra $\mathbb{T}$.

By combining lemma 6.2 with theorem 2.2, one obtains that the classical modular form $f_{E}$ attached to $E$ corresponds to a form $f$ on $\left(\mathcal{T}_{p} \times \mathcal{H}_{q}\right) / \Gamma$. The form $f$ is determined up to sign by the requirement that $f_{e^{o}}$ be normalized as in section 2 .

Let $\mathcal{H}_{q}^{\mathrm{nr}}$ be the unramified $q$-adic upper half plane defined in section 2 . Given $x_{1}$ and $x_{2}$ in $\mathcal{H}_{q}^{\mathrm{nr}}$, reducing to $v_{1}$ and $v_{2}$ respectively, define a $\mathbb{Z}$-valued harmonic cocycle on $\mathcal{E}\left(\mathcal{T}_{p}\right)$ by the rule

$$
\kappa_{f}\left\{x_{1} \rightarrow x_{2}\right\}(e)=\sum_{\epsilon: v_{1} \rightarrow v_{2}} \kappa_{f_{e}}(\epsilon)
$$

where $\kappa_{f_{e}}$ denotes the $\Gamma_{e}$-invariant harmonic cocycle attached to the $q$-adic modular form $f_{e}$ as in section 2, and the sum is taken over the set of edges $\epsilon \in \mathcal{E}\left(\mathcal{T}_{q}\right)$ in the path joining $v_{1}$ to $v_{2}$. Write $\mu_{f}\left\{x_{1} \rightarrow x_{2}\right\}$ for the measure on $\mathbb{P}_{1}\left(\mathbb{Q}_{p}\right)$ attached to $\kappa_{f}\left\{x_{1} \rightarrow x_{2}\right\}$, defined on the compact open sets $U_{e}$ by the equality

$$
\int_{U_{e}} d \mu_{f}\left\{x_{1} \rightarrow x_{2}\right\}(t)=\kappa_{f}\left\{x_{1} \rightarrow x_{2}\right\}(e) .
$$


Let $\tau_{1}$ and $\tau_{2}$ be points in $\mathcal{H}_{p}$. The next definition is motivated by the use of Teitelbaum's theorem in section 2: consider in particular formula (13). (See also the definitions of section 4 and 5.)

Definition 6.3 Define the double integral

$$
\oint_{\tau_{1}}^{\tau_{2}} \int_{x_{1}}^{x_{2}} \omega_{f}=\oint_{\mathbb{P}_{1}\left(\mathbb{Q}_{p}\right)}\left(\frac{t-\tau_{2}}{t-\tau_{1}}\right) d \mu_{f}\left\{x_{1} \rightarrow x_{2}\right\}(t) \in \mathbb{C}_{p}^{\times}
$$

Formula (59) should be regarded as the definition of the double definite integral of a "form $\omega_{f}$ " on $\mathcal{H}_{p} \times \mathcal{H}_{q}$. Such an integral can be defined solely in terms of the system of $p$-adic residues of $\omega_{f}$, which is described in definition 6.1.

Consider the assignment $(e, \epsilon) \mapsto \kappa_{f_{e}}(\epsilon)$ : it defines a $\Gamma$-invariant harmonic cocycle

$$
\lambda_{f}: \mathcal{E}\left(\mathcal{T}_{p}\right) \times \mathcal{E}\left(\mathcal{T}_{q}\right) \rightarrow \mathbb{Z}
$$

Note that $\lambda_{f}$ completely determines the form $f$. More generally, the following statement holds. Let $S_{2}\left(\left(\mathcal{T}_{p} \times \mathcal{T}_{q}\right) / \Gamma\right)_{R}$ denote the module of $\Gamma$-invariant harmonic cocycles on $\mathcal{E}\left(\mathcal{T}_{p}\right) \times \mathcal{E}\left(\mathcal{T}_{q}\right)$ with values in a ring $R$.

Lemma 6.4 The map from $S_{2}\left(\left(\mathcal{T}_{p} \times \mathcal{H}_{q}\right) / \Gamma\right)$ to $S_{2}\left(\left(\mathcal{T}_{p} \times \mathcal{T}_{q}\right) / \Gamma\right)_{\mathbb{C}_{q}}$, sending a form on $\left(\mathcal{T}_{p} \times \mathcal{H}_{q}\right) / \Gamma$ to a $\mathbb{C}_{q}$-valued harmonic cocycle on $\left(\mathcal{T}_{p} \times \mathcal{T}_{q}\right) / \Gamma$ via the natural residue map, is a Hecke-equivariant isomorphism of $\mathbb{C}_{q}$-vector spaces.

Proof: It follows from lemma 6.2 combined with the theory of residues explained in section 2 .

By interchanging the role of $p$ and $q$, one defines the concept of a weight two cusp form

$$
g: \mathcal{H}_{p} \times \mathcal{E}\left(\mathcal{T}_{q}\right) \longrightarrow \mathbb{C}_{p}
$$

on $\left(\mathcal{H}_{p} \times \mathcal{T}_{q}\right) / \Gamma$. Let

$$
\lambda_{g}: \mathcal{E}\left(\mathcal{T}_{p}\right) \times \mathcal{E}\left(\mathcal{T}_{q}\right) \rightarrow \mathbb{C}_{p}
$$

denote the $\Gamma$-invariant harmonic cocycle attached to $g$ by the rule $(e, \epsilon) \mapsto$ $\kappa_{g_{\epsilon}}(e)$.

Corollary 6.5 There exists a weight two cusp form $f^{\sharp}$ on $\left(\mathcal{H}_{p} \times \mathcal{T}_{q}\right) / \Gamma$ such that $\lambda_{f}=\lambda_{f^{\sharp}}$ (where $f$ is the weight two cusp form on $\left(\mathcal{T}_{p} \times \mathcal{H}_{q}\right) / \Gamma$ attached to the elliptic curve $E$ ). 
Proof: Use lemma 6.4 together with the fact that $\lambda_{f}$ is $\mathbb{Z}$-valued.

In the sequel of this section, denote by $f^{\sharp}$ the form satisfying the statement of corollary 6.5 .

Let $\tau_{1}$ and $\tau_{2}$ be elements in $\mathcal{H}_{p}$, and let $x_{1}$ and $x_{2}$ be elements in $\mathcal{H}_{q}^{\mathrm{nr}}$, reducing respectively to vertices $v_{1}$ and $v_{2}$ in $\mathcal{V}\left(\mathcal{T}_{q}\right)$.

Definition 6.6 Define the double integral

$$
\int_{x_{1}}^{x_{2}} \oint_{\tau_{1}}^{\tau_{2}} \omega_{f^{\sharp}}=\prod_{\epsilon: v_{1} \rightarrow v_{2}} \oint_{\tau_{1}}^{\tau_{2}} f_{\epsilon}^{\sharp}(z) d z \in \mathbb{C}_{p}^{\times} .
$$

Remark: Note that definition 6.6 is the (multiplicative) analogue in the current setting of the definition of double integral given in section 4, whereas definition 6.3 is the analogue of the definition of double integral given in section 5 . In the settings of section 4 and 5, only one way of defining the double integral was available. The next result shows that equation (60) yields the same result as (59), so that one can use definition (60) in the arguments of the remaining part of this section.

Proposition 6.7 (Fubini theorem) The equality

$$
f_{\tau_{1}}^{\tau_{2}} \int_{x_{1}}^{x_{2}} \omega_{f}=\int_{x_{1}}^{x_{2}} \mho_{\tau_{1}}^{\tau_{2}} \omega_{f}
$$

holds.

Proof: By combining definition 6.6 with Teitelbaum's theorem 2.4, one obtains

$$
\int_{x_{1}}^{x_{2}} \oint_{\tau_{1}}^{\tau_{2}} \omega_{f^{\sharp}}=\prod_{\epsilon: v_{1} \rightarrow v_{2}} \oint_{\mathbb{P}_{1}\left(\mathbb{Q}_{p}\right)} \frac{z-\tau_{2}}{z-\tau_{1}} d \mu_{f_{\epsilon}^{\sharp}}(z) .
$$

In view of definition 6.3 and equation (61), it suffices to show that

$$
d \mu_{f}\left\{x_{1} \rightarrow x_{2}\right\}=\sum_{\epsilon: v_{1} \rightarrow v_{2}} d \mu_{f_{\epsilon}^{\sharp}}
$$

By equation (58), (62) reduces to the equality

$$
\kappa_{f_{e}}(\epsilon)=\kappa_{f_{\epsilon}^{\sharp}}(e),
$$

which follows from corollary 6.5. 
In order to define an analogue of the period integral $J_{\Psi}$ considered in sections 4 and 5, one may perform cohomological manipulations similar to those of these previous sections. The exposition here follows closely the arguments of section 4 , so that only the relevant changes are explicitly mentioned. Let $q_{T}$ be the $p$-adic Tate period of $E$ (denoted by $q$ in the previous sections).

Definition 6.8 For $\tau \in \mathcal{H}_{p}$ and $x, y \in \mathcal{H}_{q}^{\mathrm{nr}}$, define the semi-indefinite double integral

$$
\int_{x}^{y} \int^{\tau} \omega_{f^{\sharp}}:=\prod_{\epsilon: r(x) \rightarrow r(y)} \oint^{\tau} f_{\epsilon}^{\sharp}(z) d z \in \mathbb{C}_{p}^{\times} / q_{T}^{\mathbb{Z}},
$$

where the semi-indefinite integral in the right-hand side of definition 6.8 is defined in section 2.

Recall from equation (21) that this definition involves a slight modification of the multiplicative definite integral.

Let $\tilde{d}_{\tau, x} \in Z^{2}\left(\Gamma, \mathbb{C}_{p}^{\times}\right)$be the two-cocycle obtained by choosing base points $\tau \in \mathcal{H}_{p}$ and $x \in \mathcal{H}_{q}^{\mathrm{nr}}$, and setting

$$
\tilde{d}_{\tau, x}\left(\gamma_{1}, \gamma_{2}\right):=\oiint_{\tau}^{\gamma_{1} \tau} \int_{\gamma_{1} x}^{\gamma_{1} \gamma_{2} x} \omega_{f}=\int_{\gamma_{1} x}^{\gamma_{1} \gamma_{2} x} \varkappa_{\tau}^{\gamma_{1} \tau} \omega_{f^{\sharp}} .
$$

The cohomology class $d \in H^{2}\left(\Gamma, \mathbb{C}_{p}^{\times}\right)$of $\tilde{d}_{\tau, x}$ does not depend on the choices of base points $\tau$ and $x$.

Proposition 6.9 The natural image of $\tilde{d}_{\tau, x}$ in $H^{2}\left(\Gamma, \mathbb{C}_{p}^{\times} / q_{T}^{\mathbb{Z}}\right)$ is zero.

Proof: Define a one-cochain $\xi_{\tau, x}: \Gamma \rightarrow \mathbb{C}_{p}^{\times} / q_{T}^{\mathbb{Z}}$ by the rule

$$
\xi_{\tau, x}(\gamma):=\int_{x}^{\gamma x} \int^{\tau} \omega_{f^{\sharp}}
$$

By a direct computation similar to that of proposition 4.3, one finds that $d \xi_{\tau, x}\left(\gamma_{1}, \gamma_{2}\right)$ is equal to the image modulo $q_{T}^{\mathbb{Z}}$ of $\tilde{d}_{\tau, x}\left(\gamma_{1}, \gamma_{2}\right)$.

Remarks:

1. In the current setting, the analogue of the module of $M$-symbols studied in section 5 is defined as follows. If $A$ is any abelian group, a function 
$m\{\rightarrow\}: \mathcal{H}_{q}^{\mathrm{nr}} \times \mathcal{H}_{q}^{\mathrm{nr}} \longrightarrow A$ denoted $(x, y) \mapsto m\{x \rightarrow y\}$ is called an $(A$ valued) $M$-symbol if it factors through the reduction map $r: \mathcal{H}_{q}^{\mathrm{nr}} \rightarrow \mathcal{V}\left(\mathcal{T}_{q}\right)$ (so that it depends only on the images of $x$ and $y$ in $\mathcal{V}\left(\mathcal{T}_{q}\right)$ ) and satisfies

$$
m\{x \rightarrow y\}+m\{y \rightarrow z\}=m\{x \rightarrow z\},
$$

for all $x, y, z \in \mathcal{H}_{q}^{\mathrm{nr}}$. Let $\mathcal{M}(A)$ denote the left $\Gamma$-module of $A$-valued $M$ symbols on $\mathcal{H}_{q}^{\mathrm{nr}}$. If one fixes $\tau \in \mathcal{H}_{p}$ and defines

$$
\tilde{c}_{\tau}(\gamma)\{x \rightarrow y\}:=\mathcal{\star}_{\tau}^{\gamma \tau} \int_{x}^{y} \omega_{f},
$$

then $\tilde{c}_{\tau}$ is an $\mathcal{M}\left(\mathbb{C}_{p}^{\times}\right)$-valued one-cocycle whose class $c \in H^{1}\left(\Gamma, \mathcal{M}\left(\mathbb{C}_{p}^{\times}\right)\right)$is independent of the choice of $\tau$. A direct computation shows that $c$ satisfies

$$
\delta(c)=d,
$$

where $\delta$ is the natural coboundary map $H^{1}\left(\Gamma, \mathcal{M}\left(\mathbb{C}_{p}^{\times}\right)\right) \longrightarrow H^{2}\left(\Gamma, \mathbb{C}_{p}^{\times}\right)$.

2. The proof of the triviality modulo $q_{T}^{\mathbb{Z}}$ of the cohomology class $d$ (and also of $c$ ) given in proposition 6.9 is based crucially on the Fubini theorem of proposition 6.7, which allows to imitate the arguments of section 4. An alternate strategy consists in using directly definition 6.3 of the double integral, and imitating the arguments of section 5. This method can be carried out, although it turns out to be less elementary. It leads to the study of the relation between $\log _{p}(c)$ and $\operatorname{ord}_{p}(c)$, which requires in particular the results of Vatsal (see [Va1] and [Va2]) on the non-vanishing of certain combinations of Gross points.

\section{The period integral}

Let $K / \mathbb{Q}$ be a quadratic algebra, and assume that it satisfies the following modified Heegner hypothesis:

1. $K$ is an imaginary quadratic field;

2. all the primes dividing $N^{-}$are inert in $K$;

3. all the primes dividing $N^{+}$are split in $K$;

4. $p$ is inert in $K$; 
5. $q$ is split in $K$.

Let $\mathcal{O}$ be a $\mathbb{Z}[1 / p q]$-order order of $K$ of conductor prime to $N$, and let $R$ be the Eichler $\mathbb{Z}[1 / p q]$-order $R$ of level $N^{+}$fixed at the beginning of this section. The above conditions ensure the existence of an oriented optimal embedding

$$
\Psi: \mathcal{O} \rightarrow R
$$

(The notions of optimality and orientation are derived by adapting those that were introduced in sections 2 and 4.)

Write $K_{p}$ and $K_{q}$ for the local algebras $K \otimes \mathbb{Q}_{p}$ and $K \otimes \mathbb{Q}_{q}$, respectively. The torus $K_{p}^{\times}$acting on $\mathcal{H}_{p}$ via the embedding $\iota \Psi$ has a unique fixed point $\tau_{\Psi}$ normalized as in equation (26). The point $\tau_{\Psi}$ belongs to $K_{p}$. Furthermore, let $\gamma_{\Psi} \in \Gamma$ be the image by $\iota \Psi$ of a generator modulo torsion of the group $\mathcal{O}_{1}^{\times}$of norm 1 elements in $\mathcal{O}^{\times}$.

Definition 6.10 Fix an element $x$ in $\mathcal{H}_{q}^{\mathrm{nr}}$. Let $\xi_{\tau, x}$ be the one-cochain defined in equation (64). The (multiplicative) period integral associated to the embedding $\Psi$ is defined to be

$$
J_{\Psi}:=\xi_{\tau_{\Psi}, x}\left(\gamma_{\Psi}\right)=\int_{x}^{\gamma_{\Psi} x} \overbrace{}^{\tau_{\Psi}} \omega_{f^{\sharp}} \in \mathbb{C}_{p}^{\times} / q_{T}^{\mathbb{Z}} .
$$

Lemma 6.11 The period $J_{\Psi}$ belongs to $K_{p}^{\times} / q_{T}^{\mathbb{Z}}$. Moreover, $J_{\Psi}$ depends only on the $\Gamma$-conjugacy class of $\Psi$, and not on the choice of $x$ that was made to define it.

Proof: The first statement follows from the calculation in the proof of theorem 6.13 below. The second statement is proved by mimicking the arguments of the previous sections.

The period $J_{\Psi}$ is related to the period

$$
I_{\Psi}:=\oint_{\bar{\tau}_{\Psi}}^{\tau_{\Psi}} \int_{x}^{\gamma_{\Psi} x} \omega_{f} \in \mathbb{C}_{p}^{\times}
$$

by the relation $J_{\Psi} / \bar{J}_{\Psi}=I_{\Psi}\left(\bmod q_{T}^{\mathbb{Z}}\right)$.

Let $w_{q}$, respectively, $w_{p}$, be equal to 1 if $E$ has split multiplicative reduction at $q$, respectively, at $p$, and let $w_{q}$, respectively, $w_{p}$ be equal to -1 otherwise. Write $\mathcal{O}_{0}$ for the maximal $\mathbb{Z}[1 / p]$-order contained in $\mathcal{O}, \mathfrak{q}$ for the class in $\operatorname{Pic}\left(\mathcal{O}_{0}\right)$ of a prime above $q$, and $h$ for the order of $\mathfrak{q}$ in $\operatorname{Pic}\left(\mathcal{O}_{0}\right)$. 
Lemma 6.12 If $w_{q}$ is equal to -1 and $h$ is odd, then $J_{\Psi}$ is equal to \pm 1 .

Proof: Let $v$ be an element of $\mathcal{O}_{0}$ whose norm is equal to $u q^{h}$ for a unit $u$ in the ring of integers of $K$, and let $\delta_{\Psi}$ be the image of $v$ by $\iota \Psi$. A direct calculation shows that

$$
\int_{\delta_{\Psi} x}^{\delta_{\Psi} \gamma_{\Psi} x} \int^{\delta_{\Psi} \tau_{\Psi}} \omega_{f^{\sharp}}=\left(\int_{x}^{\gamma_{\Psi} x} \rtimes^{\tau_{\Psi}} \omega_{f^{\sharp}}\right)^{w_{q}^{h}} .
$$

On the other hand, one has

$$
\int_{\delta_{\Psi} x}^{\delta_{\Psi} \gamma_{\Psi} x} \oiiint^{\delta_{\Psi} \tau_{\Psi}} \omega_{f^{\sharp}}=\int_{\delta_{\Psi} x}^{\gamma_{\Psi} \delta_{\Psi} x} \oiiint^{\tau_{\Psi}} \omega_{f^{\sharp}} .
$$

The claim follows by combining (65) and (66) with the second part of lemma 6.11 .

The next theorem states that $J_{\Psi}$ defines a global point on the elliptic curve $E$. In view of lemma 6.12 , assume from now that $w_{q}=1$ when $h$ is odd. Let $H_{0}$ denote the ring class field of the order $\mathcal{O}_{0}$, and let $\sigma_{\mathfrak{q}}$ be the element of $\operatorname{Gal}\left(H_{0} / K\right)$ corresponding to $\mathfrak{q}$ by the reciprocity map. Write $H$ for the subfield of $H_{0}$ which is fixed by $\sigma_{\mathfrak{q}}^{2}$. (Thus, when $h$ is odd, $H$ is simply the ring class field of the order $\mathcal{O}$.) Recall the element $\sigma_{\mathfrak{p}} \in \operatorname{Gal}\left(H_{0} / \mathbb{Q}\right)$ appearing in the statement of theorem 2.9 .

Theorem 6.13 The point $\eta_{p}\left(J_{\Psi}\right)$ is a global point $P_{\Psi}$ in $E(H)$, on which the involution $\sigma_{\mathfrak{q}}$ acts via $w_{q}$. In particular $\eta_{p}\left(I_{\Psi}\right)$ is equal to $P_{\Psi}-w_{p} \sigma_{\mathfrak{p}} P_{\Psi}$. Furthermore, for all $\mathfrak{a} \in \operatorname{Pic}^{+}(\mathcal{O})$,

$$
\eta_{p}\left(J_{\Psi^{\mathfrak{a}}}\right)=\operatorname{rec}(\mathfrak{a})^{-1} \cdot \eta_{p}\left(J_{\Psi}\right)
$$

Remark: Note the stringent analogy between theorems 2.9 and 6.13. In fact, the proof of theorem 6.13 reduces to theorem 2.9 .

Proof: By definition 6.8, one has

$$
J_{\Psi}=\prod_{\epsilon: v \rightarrow \gamma_{\Psi} v} \oint^{\tau_{\Psi}} f_{\epsilon}^{\sharp}(z) d z .
$$

Let $R_{0} \subset R$ be an Eichler $\mathbb{Z}[1 / p]$-order of level $N^{+} q$ in $B$. Denote by

$$
\Psi_{0}^{i}, \quad i=1, \ldots, h
$$


a full set of representatives for the conjugacy classes of oriented optimal embeddings from $\mathcal{O}_{0}$ to $R_{0}$ which give rise, by extension of scalars, to the conjugacy class of $\Psi$. Note that the subgroup of $\operatorname{Pic}\left(\mathcal{O}_{0}\right)$ generated by $\mathfrak{q}$ permutes simply transitively the classes of the embeddings $\Psi_{0}^{i}$. Let $\tau_{\Psi_{0}^{i}} \in K_{p}$ be the normalized fixed point for the action of $K_{p}^{\times}$on $\mathcal{H}_{p}$ via $\iota \Psi_{0}^{i}$. Let $f_{o}$ be the rigid analytic modular form on $\mathcal{H}_{p}$ corresponding to $f^{\sharp}$. By a calculation similar to the proof of proposition 4.7, one finds that if $h$ is even, the right hand side of (67) can be written as

$$
J_{\Psi}=\prod_{i=1}^{h}\left(\varkappa^{\tau_{\Psi_{0}^{i}}} f_{o}(z) d z\right)^{w_{q}^{i}} .
$$

Likewise, if $h$ is odd (so that $w_{q}=1$ by our assumptions) one finds the formula

$$
J_{\Psi}=\prod_{i=1}^{h}\left(\oint^{\tau_{\Psi_{0}^{i}}} f_{o}(z) d z\right)^{2} .
$$

But the factors

$$
\Varangle^{\tau_{\Psi_{0}^{i}}} f_{o}(z) d z
$$

appearing in (68) and (69) are the $p$-adic period integrals $J_{\Psi_{0}^{i}}$ defined in section 2. Hence the statement about $J_{\Psi}$ (including the description of the Galois action) is a consequence of the analogous statement about the $J_{\Psi_{0}^{i}}$ contained in theorem 2.9 .

\section{Periods of Hilbert modular forms}

Let $E$ be an elliptic curve defined this time over a real quadratic field $F$. It is assumed for notational simplicity (to allow ourselves to continue to use classical instead of adelic language, and thereby stress more strongly the parallel with the constructions of the previous sections) that $F$ has narrow class number one. In particular, the conductor of $E$ over $F$ is generated by a totally positive element $N$ of $\mathcal{O}_{F}$.

Choose an ordering $v_{1}, v_{2}$ of the real embeddings of $F$, and given $a \in F$, write $a_{j}$ for $v_{j}(a)$. Given an integral element $n$ of $\mathcal{O}_{F}$, denote by $|n|:=n_{1} n_{2}$ its norm, and denote likewise by $|n|$ the norm of an integral or fractional ideal of $F$. 
For each prime ideal $p$ of $F$, the integer $a_{p}$ is associated to $E$ just as in the case where $E$ is defined over $\mathbb{Q}$. Let

$$
L(E, s)=\prod_{p\lfloor N}\left(1-a_{p}|p|^{-s}+|p|^{1-2 s}\right)^{-1} \prod_{p \mid N}\left(1-a_{p}|p|^{-s}\right)^{-1}=\sum_{n} a_{n}|n|^{-s}
$$

be the Hasse-Weil L-function attached to $E / F$, where the product (resp. the sum) is taken over the prime (resp. all) ideals of $\mathcal{O}_{F}$.

Let $\Gamma \subset \mathbf{S L}_{2}\left(\mathcal{O}_{F}\right)$ be the group of matrices which are upper-triangular modulo $N$. It acts on the Poincaré upper half plane in two different ways via the embeddings $v_{1}$ and $v_{2}$, and the induced diagonal action of $\Gamma$ on $\mathcal{H} \times \mathcal{H}$ is discrete. A Hilbert modular form of weight $(2,2)$ and level $N$ is a holomorphic function $f\left(\tau_{1}, \tau_{2}\right)$ on $\mathcal{H} \times \mathcal{H}$ satisfying

1. The differential two-form $\omega:=f\left(\tau_{1}, \tau_{2}\right) d \tau_{1} d \tau_{2}$ is invariant under $\Gamma$;

2. The form $\omega$ is holomorphic at the cusps.

The analogue of the Shimura-Taniyama conjecture in this setting predicts that the holomorphic function on $\mathcal{H} \times \mathcal{H}$ given by the absolutely convergent Fourier series

$$
f\left(\tau_{1}, \tau_{2}\right)=\sum_{n \gg 0} a_{(n)} e^{2 \pi i\left(\frac{n_{1}}{d_{1}} \tau_{1}+\frac{n_{2}}{d_{2}} \tau_{2}\right)},
$$

is a Hilbert modular form of weight $(2,2)$ and level $N$. Here the sum is taken over all totally positive elements of $\mathcal{O}_{F}$, and $d$ is a totally positive generator of the different ideal of $\mathcal{O}_{F}$. Let $\varepsilon$ be a unit of $\mathcal{O}_{F}$, such that $\varepsilon_{1}<0$ and $\varepsilon_{2}>0$, and define

$$
\begin{array}{ll}
\omega_{++}=-4 \pi^{2} f\left(\tau_{1}, \tau_{2}\right) d \tau_{1} d \tau_{2}, & \omega_{-+}=4 \pi^{2} f\left(\varepsilon_{1} \bar{\tau}_{1}, \varepsilon_{2} \tau_{2}\right) d \bar{\tau}_{1} d \tau_{2}, \\
\omega_{+-}=4 \pi^{2} f\left(-\varepsilon_{1} \tau_{1},-\varepsilon_{2} \bar{\tau}_{2}\right) d \tau_{1} d \bar{\tau}_{2}, & \omega_{--}=-4 \pi^{2} f\left(-\bar{\tau}_{1},-\bar{\tau}_{2}\right) d \bar{\tau}_{1} d \bar{\tau}_{2} .
\end{array}
$$

Assume that $E$ satisfies the conclusion of the Shimura-Taniyama conjecture. The periods attached to $\omega$ have been much studied classically and can be computed numerically in practice from the Fourier expansion of equation (70). Define a $\Gamma$-invariant $\mathbb{R}$-valued double integral on $\mathcal{H} \times \mathcal{H}$ by the rule:

$$
\int_{\tau_{1}}^{\tau_{2}} \int_{\tau_{3}}^{\tau_{4}} \omega_{f}:=\int_{\tau_{1}}^{\tau_{2}} \int_{\tau_{3}}^{\tau_{4}}\left(\omega_{++}+\omega_{+-}+\omega_{-+}+\omega_{--}\right)
$$

where the integral on the right is the usual complex integral which can be computed using the Fourier series expansion (70) for $f$. 
With this integral in hand, we can mimick the definitions in sections 4 , 5 , and 6 , letting $\tilde{d}_{\tau, x} \in Z^{2}(\Gamma, \mathbb{R})$ be the two-cocycle obtained as in equation (36) by choosing base points $\tau, x \in \mathcal{H}$ and setting

$$
\tilde{d}_{\tau, x}\left(\gamma_{1}, \gamma_{2}\right):=\int_{\tau}^{\gamma_{1} \tau} \int_{\gamma_{1} x}^{\gamma_{1} \gamma_{2} x} \omega_{f}
$$

Let $d \in H^{2}(\Gamma, \mathbb{R})$ be the cohomology class of $\tilde{d}_{\tau, x}$. It does not depend on the choices of base points $\tau$ and $x$ that were made to define $\tilde{d}_{\tau, x}$.

If one fixes $\tau \in \mathcal{H}$ and defines, for $x, y \in \mathbb{P}_{1}(F)$ any cusps on the Hilbert modular surface:

$$
\tilde{c}_{\tau}(\gamma)\{x \rightarrow y\}:=\int_{\tau}^{\gamma \tau} \int_{x}^{y} \omega_{f}
$$

then $\tilde{c}_{\tau}$ is an $\mathcal{M}(\mathbb{R})$-valued one-cocycle, whose image $c$ in $H^{1}(\Gamma, \mathcal{M}(\mathbb{R}))$ is related to the 2 -cocycle $\tilde{d}_{\tau, x}$ as in section 5 :

$$
\delta(c)=d .
$$

Choose a Néron differential $\omega_{E}$ for $E / F$, and let $\Omega_{1}$ and $\Omega_{2}$ be the real periods attached to this choice. Note that $\omega_{E}$ is only well-defined up to multiplication by a power of $\varepsilon$, so that $\Omega_{1}$ and $\Omega_{2}$ are only well-defined up to multiplication by a power of $\varepsilon_{1}$ and $\varepsilon_{2}$ respectively. But the product $\Omega_{1} \Omega_{2}$ is well-defined up to sign.

The following conjecture, referred to from now on as the period conjecture, is a natural analogue of proposition 4.3 of section 4 , theorem 5.2 of section 5 , and proposition 6.9 of section 6 .

Conjecture 7.1 There exists a lattice $\Lambda \subset \mathbb{R}$ which is commensurable to the lattice generated by $\Omega_{1} \Omega_{2}$, and such that the natural images of $d$ in $H^{2}(\Gamma, \mathbb{R} / \Lambda)$ and of $c$ in $H^{1}(\Gamma, \mathcal{M}(\mathbb{R} / \Lambda))$ are trivial.

Remark. Conjecture 7.1 predicts in particular that if $\alpha / \nu \in \mathbb{P}_{1}(F)$ is a cusp (with $\alpha, \nu \in \mathcal{O}_{F}$ and $\operatorname{gcd}(\alpha, \nu)=1$ ) and $\gamma=\gamma_{\alpha / \nu} \in \Gamma_{0}(N)$ is a generator for the stabiliser of $\alpha / \nu$ and $\infty$, then the period

$$
J_{\alpha / \nu}:=\tilde{c}_{\tau}(\gamma)\{\infty \rightarrow \alpha / \nu\}=\int_{\tau}^{\gamma \tau} \int_{\alpha / \nu}^{\infty} \omega_{f} \text { belongs to } \Lambda \text {. }
$$

This is consistent with (a special case of) the Birch and Swinnerton-Dyer conjecture for the $L$-function of $E / F$ and its twists by abelian characters of 
$F$. Recall that if $\chi: \operatorname{Gal}(\bar{F} / F) \longrightarrow \mathbb{C}^{\times}$is a finite order character, the twisted $L$-series is defined in the usual way by the rule

$$
L(E / F, \chi, s)=\sum_{n} \chi(n) a_{n}|n|^{-s} .
$$

A direct calculation shows that the integral on the right of (73) is equal to the integral of $\omega_{f}$ on the cycle denoted $\gamma_{\alpha / \nu, E}$ in $\S 15$ of [Oda]. (In Oda's notation $E$ denotes the subgroup of $\mathcal{O}_{F}^{\times}$generated by the eigenvalues of $\gamma$.)

Let $c_{1}$ and $c_{2}$ denote the conjugacy classes of complex conjugation attached to the infinite places $v_{1}$ and $v_{2}$ respectively. (These are the "Frobenius elements at $\infty$ " attached to the real places of $F$.) A character $\chi$ of $\left.G_{F}=\operatorname{Gal} \bar{F} / F\right)$ is called even if $\chi\left(c_{1}\right)=\chi\left(c_{2}\right)=1$, so that $\chi$ factors through the Galois group of a totally real abelian extension of $F$.

Theorem 16.3 (combined with lemma 15.4) of [Oda] asserts (in the special case where $N=1$, but the calculations explained there are readily adapted to more general $N$ ) that for all characters $\chi:\left(\mathcal{O}_{F} / \nu\right)^{\times} /\left(\mathcal{O}_{F}^{\times 2}\right) \longrightarrow \mathbb{C}^{\times}$(which can be viewed as characters of $G_{F}$ in the usual way)

$$
\sum_{\alpha \in \mathcal{O}_{F} / \nu} \chi(\alpha) J_{\alpha / \nu} \doteq \begin{cases}G(\bar{\chi}) L(E / F, \chi, 1) & \text { if } \chi \text { is even } \\ 0 & \text { otherwise }\end{cases}
$$

where $G(\bar{\chi})$ is a Gauss sum and where the symbol $\doteq$ denotes equality up to rational multiples with denominator bounded independently of $\chi$ and $\nu$. On the other hand, a natural equivariant extension of the Birch and SwinnertonDyer conjecture predicts that for all even characters $\chi$ the ratios

$$
G(\bar{\chi}) L(E / F, \chi, 1) /\left(\Omega_{1} \Omega_{2}\right)
$$

are algebraic (in the field generated by the values of $\chi$, and with denominators bounded independently of $\chi$ ). Thus the Birch and Swinnerton-Dyer conjecture leads one to expect the vanishing of all the periods $J_{\alpha / \nu}$ modulo a lattice $\Lambda \subset \mathbb{R}$ which is commensurable with $\mathbb{Z} \cdot \Omega_{1} \Omega_{2}$.

Assume, at the cost of eventually multiplying $\tilde{c}_{\tau}$ and $\tilde{d}_{\tau, x}$ by a common integer, that the lattice $\Lambda$ of conjecture 7.1 can be chosen to be equal to $\mathbb{Z} \cdot \Omega_{1} \Omega_{2}$. Thanks to conjecture 7.1 , we may then define an $M$-symbol $m_{\tau} \in$ $C^{0}\left(\Gamma, \mathcal{M}\left(\mathbb{R} / \mathbb{Z} \cdot \Omega_{1} \Omega_{2}\right)\right)$ and a one-cochain $\xi_{\tau, x} \in C^{1}\left(\Gamma, \mathbb{R} / \mathbb{Z} \cdot \Omega_{1} \Omega_{2}\right)$ by the rules

$$
\tilde{c}_{\tau}=d m_{\tau}, \quad \tilde{d}_{\tau, x}=d \xi_{\tau, x}
$$


We adopt as before the useful notation

$$
\int^{\tau} \int_{x}^{y} \omega_{f}:=m_{\tau}\{x \rightarrow y\} \in \mathbb{R} / \mathbb{Z} \cdot \Omega_{1} \Omega_{2} .
$$

Let $K$ be a quadratic extension of $F$, which is imaginary at $v_{1}$ and real at $v_{2}$. (Thus, in particular, $K$ is not itself Galois over $\mathbb{Q}$.) Let $\mathcal{O}$ be the ring of integers of $K$ (or a more general $\mathcal{O}_{F}$-order in $K$ ). The notion of an oriented optimal embedding $\Psi: K \longrightarrow M_{2}(F)$ is defined exactly as in the previous sections. One can then associate to any oriented optimal embedding $\Psi$ a period by letting $\tau \in \mathcal{H}_{1}$ be the unique fixed point of $\Psi\left(K^{\times}\right)$acting on $\mathcal{H}_{1}$, letting $\gamma_{\tau}=\Psi(\epsilon) \in \Gamma$, and setting

$$
J_{\Psi}:=\xi_{\tau, x}\left(\gamma_{\tau}\right)=\int^{\tau} \int_{x}^{\gamma_{\tau} x} \omega_{f} \in \mathbb{R} / \mathbb{Z} \cdot \Omega_{1} \Omega_{2},
$$

for any $x \in \mathbb{P}_{1}(F)$. As in the previous discussions, the period $J_{\Psi}$ depends only on the $\Gamma$-conjugacy class of $\tau$ (and therefore, of $\Psi$ ), not on the choices of $x$ and then $\xi_{\tau, x}$ that were made to define it. This follows from manipulations identical to those that were used to prove the corresponding statement in section 4.

Let $H$ denote the ring class field of $K$ attached to the order $\mathcal{O}$. The reciprocity map of class field theory gives a canonical identification

$$
\text { rec }: \operatorname{Pic}(\mathcal{O}) \longrightarrow \operatorname{Gal}(H / K) \text {. }
$$

Let $\eta$ be the Weierstrass uniformisation of $E_{1}$ over $\mathbb{R}$, associated to the differential $\omega_{E}$ that was chosen earlier in defining the periods $\Omega_{1}$ and $\Omega_{2}$. For all $\Psi$ in $\operatorname{Emb}(\mathcal{O}, R)$, the period $J_{\Psi} / \Omega_{2}$ is a well defined element of $\mathbb{R} / \mathbb{Z} \cdot \Omega_{1}$ on which it is natural to compute the uniformisation $\eta$. The following conjecture is the counterpart to theorem 4.8, conjecture 5.4, and theorem 6.13 in a purely archimedean setting:

Conjecture 7.2 For all $\Psi$ in $\operatorname{Emb}(\mathcal{O}, R)$, the point $\eta\left(J_{\Psi} / \Omega_{2}\right)$ belongs to $E(H)$. More precisely, for all $\mathfrak{b} \in \operatorname{Pic}(\mathcal{O})$,

$$
\eta\left(J_{\Psi^{\mathfrak{b}}} / \Omega_{2}\right)=\operatorname{rec}(\mathfrak{b})^{-1} \cdot \eta\left(J_{\Psi} / \Omega_{2}\right) .
$$




\section{References}

[BCDT] C. Breuil; B. Conrad; F. Diamond; R. Taylor. On the modularity of elliptic curves over $\mathbf{Q}$ : wild 3-adic exercises. J. Amer. Math. Soc. 14 (2001), no. 4, 843-939.

[BD1] M. Bertolini; H.Darmon. Heegner points on Mumford-Tate curves. Invent. Math. 126 (1996), no. 3, 413-456.

[BD2] M. Bertolini; H. Darmon. Heegner points, p-adic L-functions, and the Cerednik-Drinfeld uniformisation. Invent. Math. 131 (1998), no. 3, 453-491.

[BD3] M. Bertolini; H. Darmon. The p-adic L-functions of modular elliptic curves. Mathematics unlimited-2001 and beyond, 109-170, Springer, Berlin, 2001.

[BDIS] M. Bertolini; H. Darmon, A. Iovita, and M. Spiess. Teitelbaum's exceptional zero conjecture in the anticyclotomic setting. American Journal of Mathematics 124 (2002) 411-449.

[BoCa] J.-F. Boutot; H. Carayol. Uniformisation p-adique des courbes de Shimura: les théorèmes de Cerednik et de Drinfeld. Courbes modulaires et courbes de Shimura (Orsay, 1987/1988). Astérisque No. 196197, (1991), 7, 45-158 (1992).

$[\mathrm{Bu}]$ D. Bump. Automorphic forms and representations. Cambridge Studies in Advanced Mathematics, 55. Cambridge University Press, Cambridge, 1997.

[CF] Algebraic number theory. Proceedings of an instructional conference organized by the London Mathematical Society. Edited by J. W. S. Cassels and A. Fröhlich. Academic Press, London; Thompson Book Co., Inc., Washington, D.C. 1967.

[Da] H. Darmon. Integration on $\mathcal{H}_{p} \times \mathcal{H}$ and arithmetic applications. Annals of Math 154 (2001) 589-639.

[DG] H. Darmon, P. Green. Elliptic curves and class fields of real quadratic fields: algorithms and evidence. Journal of Experimental Mathematics 11:1 (2002) 37-55. 
[GS] R. Greenberg, G. Stevens. p-adic L-functions and p-adic periods of modular forms. Invent. Math. 111 (1993), no. 2, 407-447.

[GvdP] L. Gerritzen; M. van der Put. Schottky groups and Mumford curves. Lecture Notes in Mathematics, 817. Springer, Berlin, 1980.

[I] Y. Ihara. On congruence monodromy problems. Vol. 1. Lecture Notes, No. 1. Department of Mathematics, University of Tokyo, Tokyo 1968.

[JoLi1] B.W. Jordan; R. Livné. Local diophantine properties of Shimura curves, Math. Ann. 270 (1985) 235-248.

[JoLi2] B.W. Jordan; R. Livné. Integral Hodge theory and congruences between modular forms. Duke Math. J. 80 (1995) 419-484.

[La] S. Lang, Introduction to modular forms. Grundlehren der mathematischen Wissenschaften, No. 222. Springer-Verlag, Berlin-New York, 1976 .

[Man1] Y. Manin. Parabolic points and zeta functions of modular curves. Izv. Akad. Nauk SSSR Ser. Mat. 36 (1972), 19-66.

[Man2] Y. Manin. p-adic automorphic functions. J. Sov. Math. 5 (1976) 279334.

[Maz] B. Mazur. Courbes elliptiques et symboles modulaires. Séminaire Bourbaki, 24ème année (1971/1972), Exp. No. 414, pp. 277-294. Lecture Notes in Math., Vol. 317, Springer, Berlin, 1973.

[MS] Y. Matsushima; G. Shimura. On the cohomology groups attached to certain vector valued differential forms on the product of the upper half planes. Ann. of Math. (2) 78 (1963) 417-449.

[MTT] B. Mazur; J. Tate; J. Teitelbaum. On p-adic analogues of the conjectures of Birch and Swinnerton-Dyer. Invent. Math. 84 (1986), no. 1, $1-48$.

[Oda] T. Oda. Periods of Hilbert modular surfaces. Progress in Mathematics, 19. Birkhäuser, Boston, Mass., 1982. 
[Sch] P. Schneider. Rigid-analytic L-transforms. Number theory, Noordwijkerhout 1983 (Noordwijkerhout, 1983), 216-230, Lecture Notes in Math. 1068, Springer, Berlin, 1984.

[Se1] J.-P. Serre. Complex multiplication. 1967 Algebraic Number Theory (Proc. Instructional Conf., Brighton, 1965) pp. 292-296, Thompson, Washington, D.C.

[Se2] J.-P. Serre. Le problème des groupes de congruence pour $\mathbf{S L}_{2}$. Ann. of Math. (2) 92 (1970) 489-527.

[Sh1] G. Shimura, Class-fields and automorphic functions. Ann. of Math. (2) 80 (1964) 444-463.

[Sh2] G. Shimura, Construction of class fields and zeta functions of algebraic curves. Ann. of Math. (2) 85 (1967) 58-159.

[Sh3] G. Shimura, Algebraic number fields and symplectic discontinuous groups. Ann. of Math. (2) 86 (1967) 503-592.

[Si] J. Silverman. The arithmetic of elliptic curves. Graduate Texts in Mathematics, 106. Springer-Verlag, New York, 1986.

[St] H.M. Stark. Modular forms and related objects. Number theory (Montreal, Que., 1985), 421-455, CMS Conf. Proc., 7, Amer. Math. Soc., Providence, RI, 1987.

[Te] J.T. Teitelbaum. Values of p-adic L-functions and a p-adic Poisson kernel. Invent. Math. 101 (1990), no. 2, 395-410.

[Va1] V. Vatsal. Uniform distribution of Heegner points. Invent. Math. 148 (2002) 1-46.

[Va2] V. Vatsal. Special values of anticyclotomic L-functions. preprint.

[Vi] M-F. Vignéras. Arithmétique des algèbres de quaternions. Lecture Notes in Mathematics, 800. Springer, Berlin, 1980.

[Wi] A. Wiles. Modular elliptic curves and Fermat's last theorem. Ann. of Math. (2) 141 (1995), no. 3, 443-551.

[Zh] S. Zhang. Heights of Heegner points on Shimura curves. Ann. of Math. (2) 153 (2001), no. 1, 27-147. 